PSYCHOMETRIKA-VOL. 43, NO. 4.

DECEMBER, 1978

\title{
A DIRECT APPROACH TO INDIVIDUAL DIFFERENCES SCALING USING INCREASINGLY COMPLEX TRANSFORMATIONS
}

\author{
JAMES C. LINGOES
}

THE UNIVERSITY OF MICHIGAN

INGWER BORG

\author{
RHEINISCH-WESTFÄLISCHE TECHNISCHE HOCHSCHULE
}

\begin{abstract}
A family of models for the representation and assessment of individual differences for multivariate data is embodied in a hierarchically organized and sequentially applied procedure called PINDIS. The two principal models used for directly fitting individual configurations to some common or hypothesized space are the dimensional salience and perspective models. By systematically increasing the complexity of transformations one can better determine the validities of the various models and assess the patterns and commonalities of individual differences. PINDIS sheds some new light on the interpretability and general applicability of the dimension weighting approach implemented by the commonly used INDSCAL procedure.
\end{abstract}

Key words: individual differences scaling, procrustean transformations, matrix fitting, configuration similarity.

Following Horan's [1969] original proposal for a subjective metrics model, there has been considerable effort devoted to solving the mathematical problems and to constructing computer algorithms for scaling real data [e.g.: Bloxom, Note 1; Carroll \& Chang, 1970, Note 2; Carroll \& Wish, 1974; Schönemann, 1972; Schönemann et al., Note 7; Takane et al. 1977]. Because Carroll \& Chang's IDIOSCAL procedure, and its special case INDSCAL, is by far the most well-known solution to the "dimensional salience model", we shall briefly review the general issues in this area using the INDSCAL terminology.

In the dimensional salience (or weighted euclidean) model, the distance between points $p$ and $q$ in individual $i$ 's "private perceptual space" is estimated from the respective points in some average configuration of all $N$ individuals in the sample by the following weighted euclidean distance formula:

$$
d_{p q}^{(l)} \cong d_{p q}^{(l)}=\left[\sum_{a} c_{a}^{(\ell)}\left(g_{p a}-g_{q a}\right)^{2}\right]^{1 / 2}
$$

where $d_{p q}^{(i)}$ is the distance between $p$ and $q$ (given as datum), $d_{p q}^{(i)}$ is an estimate of $d_{p q}^{(i)}$ (e.g., a least-squares estimate), $c_{a}^{(l)}$ is the squared weight associated with coordinate axis $a, g_{p a}$ is the projection of $p$ on $a$ in the $n \times m$ "group space" $G$, and the superscript $i$ indicates individual $i$. As is true for all scaling algorithms proposed so far for the dimensional salience model, INDSCAL does not attempt a direct solution for the unknowns in (1), i.e., for $c_{a}^{(i)}(a=1, \cdots, m ; i=1, \cdots, N)$ and $G$. Rather, (1) is converted into its simpler scalarproduct form: centering $G$ at the origin and using the well-known relation:

$$
b_{p Q}^{(i)}=\frac{1}{2}\left[\sum_{a} c_{a}^{(i)} g_{p a}^{2}+\sum_{a} c_{a}^{(i)} g_{q a}^{2}-d_{p q}^{(i) 2}\right] \text {, }
$$

Requests for reprints should be sent to James C. Lingoes, The University of Michigan, 1005 North University Building, Ann Arbor, Michigan 48109. 
one arrives at

$$
\hat{b}_{p q}^{(i)}=\sum_{a} g_{p a} c_{a}^{(t)} g_{q a},
$$

where $\hat{b}_{p q}^{(i)}$ is the estimated scalar product of $p$ and $q$ for individual $i$. Now, since the given distances $d_{p q}^{(i)}(p, q=1, \cdots, n ; i=1, \cdots, N)$ are determined only up to an arbitrary origin (if one assumes that they are interval scaled), one must decide on some origin in order to generate the corresponding scalar products for each individual, because INDSCAL uses, for reasons of computational simplicity, scalar products rather than distances. The estimation of a convenient origin is implemented by Torgerson's additive constant method [1958].

Equation (3) can also be expressed more compactly in matrix notation as:

$$
\hat{B}_{i}=G C_{i} G^{\prime},
$$

where $G$ is $n \times m$ and $C_{l}$ is diagonal (the INDSCAL implementation of the subjective metrics model) or is constrained to be (semi-) definite (the IDIOSCAL model with positivity constraints). An alternating least-squares (ALS) procedure is then used to solve for $G$ and $C_{t}(i=1, \cdots, N)$. In case of a non-diagonal $C_{i}$, Carroll \& Chang [Note 3] propose a further decomposition of $C_{i}$ into

$$
C_{i}=T_{i} \Lambda_{i} T^{\prime}{ }_{l}
$$

where $T_{i} T^{\prime}{ }_{i}=T^{\prime}{ }_{i} T_{i}=I$ and $\Lambda_{i}$ is diagonal. Geometrically, this corresponds to an orthogonal "idiosyncratic" rotation of $G$ by $T_{l}$, followed by dimension weighting with $\Lambda_{i}^{1 / 2}$. In contrast with the INDSCAL solution, however, in which $G$ and $C_{i}$ are not rotationally invariant, IDIOSCAL will yield results which are determined only up to a joint rotation of $G$ and $C_{t}$. Furthermore, the decomposition in (5) is obviously not the only possible one: Harshman [Note 4] and Tucker [1972] propose other, non-orthogonal factorizations.

Although INDSCAL, in particular, has been applied very successfully in various contexts, some problems associated with the IDIOSCAL family of procedures (of which INDSCAL is but a special case) remain. The most obvious of these difficulties lies in the choice of loss function: goodness of fit is defined in terms of scalar products, which are interpretationally more indirect and complicated than distances. Moreover, the transformations used to fit $G$ to each individual's data assume a relatively simple relationship between the two configurations. If some of the points therein have different relative locations or if the configurations are unevenly or nonlinearly distorted, the dimensional salience model has no means of detecting this. This could have the consequence that the computed group space is quite misleading, representing a confounding of a class of essentially qualitatively different configurations. Finally, a most serious disadvantage is that the substantive and statistical significance of the transformations is not clear. The dimension weighting, for example, might produce a quite negligible fit improvement over a classical procrustean fitting of the configurations which leaves invariant the relative distances. It is clearly desirable to disentangle the admissible transformations from those that change the meaningful properties of the group space by some distortion, a point which is to some extent taken into account in the third phase of IDIOSCAL [Carroll \& Chang, Note 3], but not at all for the more widely used INDSCAL procedure.

Note that not all algorithms developed in this area share all of INDSCAL's weaknesses. Schönemann et al.'s COSPA [Note 7] even provides some tests for the applicability of the dimensional salience model, but it also introduces some additional and fairly strong assumptions not included in the original formulations of the model. However, neither COSPA nor INDSCAL nor ALSCAL [Takane et al., 1977] minimizes a loss function 
defined for (unsquared) distances, nor do any of these procedures provide information with respect to the performance of admissible (nondistorting) transformations. Furthermore, no method adds anything to the dimensional salience model which might provide information regarding individual differences of a more complicated nature.

\section{The PINDIS Model}

PINDIS (Procrustean INdividual DIfferences Scaling: Lingoes, Note 6; Lingoes \& Borg, 1976, 1977; Borg \& Lingoes, Note 2, 1977; Borg, 1977) is similar to procedures like IDIOSCAL in the sense that PINDIS also scales individual similarities according to the dimensional salience model. It is different, however, from all other algorithms and models in a number of respects: (a) in PINDIS, the dimensional salience model is only one approach for assessing individual differences; (b) the set of models in PINDIS is organized into two hierarchies with a typical common order, i.e., the procedure sequentially analyzes the relationship of individuals to some group space in such a way that a fitting is done first by strictly admissible transformations (those that leave the relative distances invariant) and then by adding increasingly more free parameters in the form of weights, idiosyncratic rotations and translations.

Also different from other approaches in this respect, PINDIS starts with a set of individual configurations $X_{t}(i=1, \cdots, N)$ rather than with either scalar product or distance matrices. These $X_{i}$ 's are assumed to be given by some prior and assumptionappropriate analysis, either metric or nonmetric. Each $X_{i}$, an order $n \times m_{i}$ matrix, is assumed to be arbitrary insofar as only comparative distances among the $n$ points are to remain invariant, i.e., $X_{l}$ is determined up to rigid motions and central dilations. Consequently, we will always exhaust the complete set of admissible transformations, i.e., rotations/reflections, translations, and central dilations on $X_{i}$, in fitting $X_{i}$ to the target configuration at each stage of the PINDIS analysis. This tranformed $X_{i}$ will be symbolized by $\tilde{X}_{t}$ and is to be interpreted as including any or all of the permitted transformations in the group relevant to the particular target under analysis.

Initially, we arbitrarily norm all $X_{i}$ 's (centered at the origin) to unit length, which constitutes the optimal translation on $X_{i}$ for the following analyses: a centroid configuration $Z$ of order $n \times m\left(m=\max _{i}\left(m_{i}\right)\right)$, which is analogous to IDIOSCAL's group space $G$, is computed as the average of all $X_{i}$ 's after they have been fitted optimally to each other under the admissible transformations. Since $Z$ 's orientation is arbitrary, it is put into an orientation that is optimal for dimension weighting of all $X_{i}$ 's, which will be discussed in more detail below. The similarity of each $X_{i}$ to $Z$ is expressed by $r^{2}\left(X_{i}, Z\right)$, the squared product-moment correlation between the corresponding elements of $\tilde{X}_{i}$ and $Z$ [Lingoes \& Schönemann, 1974]. This is the first important index in PINDIS, representing a reference value for those transformations that do not preserve the distance ratios in $Z$. Centering $X_{i}$ is also optimal for the dimension salience model, which constitutes the next stage of analysis.

The simplest distortion of $Z$ for mapping $X_{i}$ is that of dimension weighting, where a diagonal $m$-square weight matrix $W_{i}$ is determined for each optimally transformed individual configuration $X_{i}$. Another fit index, $r^{2}\left(\tilde{X}_{i}, Z^{r} W_{i}\right)$ yields the relevant information about the explanatory power of such weighting. Obviously, one would not want to attach much psychological meaning to the differences among the elements of $W_{i}$ if $r^{2}\left(\tilde{X}_{i}, Z^{r} W_{i}\right)$, where $Z^{r}$ is the optimally rotated $Z$ for all $X_{t}$, is not substantially higher than our reference value, $r^{2}\left(\tilde{X}_{i}, Z\right)$. Similarly, one would also hesitate to interpret an idiosyncratic rotation of $Z$, at the next transformation stage of the dimensional salience model in PINDIS, as having much substantive import, if $r^{2}\left(\tilde{X}_{i}, Z^{r} W_{l}\right)$ is not appreciably smaller than $r^{2}\left(\tilde{X}_{i}\right.$, $Z_{t}^{r} W_{i}^{r}$ ), where $Z_{t}^{r}=Z S_{t}, S_{i} S_{i}^{\prime}=S_{i}^{\prime} S_{t}=I$ (the identity matrix) and $W_{i}^{r}$ is the diagonal weight matrix for $Z_{i}^{r}$. It should be noted that since we want just one "average subject" or 
one $Z$ for explaining individual differences, we consider such idiosyncratic rotations of $Z$ as being in the same class of inadmissible transformations as those of dimension weighting.

Using even more free parameters, one could now fit $V_{t} Z$ to the optimally transformed $X_{i}$, where $V_{i}$ is an $n$-square diagonal matrix of vector weights. Geometrically, the points in $Z$ are herein conceived of as termini of vectors emanating from the origin of $Z$ : the weights of $V_{l}$ will then stretch or shrink each vector with or without reversing its sense. The measure which indicates the success of such fitting is $r^{2}\left(\tilde{X}_{i}, V_{i} Z^{t}\right)$ where $Z^{t}$ is an optimally translated $Z$ for all $X_{t}$. However, it will be shown below that generally there exists an idiosyncratic translation of $Z$ relative to each $\tilde{X}_{i}$ which improves the predictability of $\tilde{X}_{i}$. The latter represents a subject-specific mapping in the context of vector weighting, analogous to the individual rotations of $Z$ in the dimension-weighting approach, and, as such, are also considered to be inadmissible along with vector weighting. The fit index for idiosyncratic translations is $r^{2}\left(X_{t}, V_{t}^{t} Z_{t}^{t}\right)$, where $Z_{i}^{t}$ is the idiosyncratically translated $Z$ and $V_{i}^{t}$ is the associated weight matrix for this mapping. Both of these fit indices for vector weighting can be compared with one another and both with our basic, non-distorting, measure of fit, $r^{2}\left(\tilde{X}_{l}, Z\right)$, for assessing the relevance of these transformations. The vector weighting model provides not only indices of inter-individual differences, but also represents a psychological model in its own right (which we call the "perspective model") for a number of psychological and social contexts.

We can now summarize the various PINDIS transformations in Table 1. Note that $X_{i}$ is defined as that $X_{i}$ which is transformed by admissible mappings in such a way that the respective fit index is maximized. Thus, it is clear that inadmissible mappings are always associated with the centroid configuration $Z$. The distinction admissible vs. inadmissible transformation is based upon the property of the former to be indeterminate from either the data itself or from the respective fitting model. Consequently, admissible transformations can have no substantive meaning whatsoever: only the listed inadmissible fitting parameters are principally interpretable.

From Table 1 one can discern two model hierarchies contained in a PINDIS anal-

TABLE 1

Summary of PINDIS Transformations

\begin{tabular}{ccc}
\hline Model & $\begin{array}{c}\text { Number of } \\
\text { inadmissible } \\
\text { fitting parameters }\end{array}$ & Fit index \\
\hline $\begin{array}{c}\text { Similarity transformation } \\
\text { (unit weighting) }\end{array}$ & 0 & $\mathbf{r}^{2}\left(\overline{\mathbf{x}}_{\mathbf{1}}, \mathbf{Z}\right)$ \\
$\begin{array}{c}\text { Dimensional salience } \\
\text { (dimension weighting) } \\
\text { Dimensional salience } \\
\text { with idiosyncratic } \\
\text { orientation }\end{array}$ & $\mathrm{m}+\left({ }_{2}\right)$ & $\mathbf{r}^{2}\left(\overline{\mathbf{x}}_{1}, \mathbf{Z}^{r} \mathbf{w}_{1}\right)$ \\
$\begin{array}{c}\text { Perspective model } \\
\text { with fixed origin } \\
\text { (vector weighting) } \\
\begin{array}{c}\text { Perspective model } \\
\text { with idiosyncratic } \\
\text { origin }\end{array}\end{array}$ & $\left.\mathrm{n}, \mathbf{Z}_{1}^{r} \mathbf{w}_{1}^{\mathrm{r}}\right)$ \\
\hline
\end{tabular}


ysis one hierarchy is established by the triple (1) similarity transformation, (2) dimensional salience with a fixed orientation, and (3) dimensional salience with idiosyncratic orientations; the other hierarchical triple is formed by (1) similarity transformation, (2) perspective model with fixed origin, and (3) perspective model with idiosyncratic origins. Both of these model collections are hierarchical in the sense that the models are nested, the one using fewer inadmissible fitting parameters being but a special case of the one using more. However, since in empirical applications one would typically encounter situations where $n$ is much greater than $m$, one would also have an order among all models established by the number of free parameters, i.e., the typical order is

$$
0<m<m+\left(\begin{array}{c}
m \\
2
\end{array}\right)<n<n+m .
$$

In a more general sense, the various PINDIS mappings belong to the group of affine transformations defined, respectively, on the column and row spaces of the various configurations with the similarity transformation being but a special case of unit weightings for both sub-models.

Such a hierarchical approach yields standards against which the significance of the inadmissible transformations can be judged. Furthermore, it provides many indices (v.i.) which not only represent additional information in their own right, but which also allow one to evaluate the validity of various features of the dimensional salience model for a given sample of individuals.

\section{The Centroid Configuration, $Z$}

The centroid configuration, $Z$, is calculated from the $X_{i}$ 's by a method similar to the one proposed by Gower [1975]. (Gower calls $Z$ the "consensus configuration", a term which carries unnecessary and potentially misleading psychological connotations. We prefer a more neutral name.)

The derivation of $Z$ starts with the individual $X_{i}$ 's, where each $X_{l}$ is an $n \times m_{l}$ coordinate matrix generated by a prior MDS, factor analysis, or any other appropriate multidimensional procedure. It is not necessary that all individual (perceptual) spaces be of the same dimensionality if one adds zero-element columns to $X_{i}$ 's whenever $m_{i}<m$. The goal is to rescale all $X_{i}$ 's simultaneously by affine transformations to $\tilde{X}_{i}=X_{i} R_{i}-j u_{i}^{\prime}$, such that the following fit criterion, representing a residual sum of squares, is optimized:

$$
L=\sum_{i}\left[\operatorname{tr}\left(X_{i}-Z\right)\left(\tilde{X}_{i}-Z\right)^{\prime}\right]
$$

where $R_{i}^{\prime} R_{i}=R_{i} R_{l}^{\prime}=I, j$ is an $n$-element vector of ones, and $u_{i}$ is an $m$-element translation vector. To minimize $L$, each $X_{i}$ is centered at the origin first, which represents the optimal $u_{i}$ motion, and then centrally contracted or dilated such that its euclidean norm, $\operatorname{tr}\left(X_{i} X_{i}^{\prime}\right)$ equals unity. We then proceed iteratively as follows:

(a) $X_{2}$ is then rotated to fit $X_{1}$ in a procrustean sense and a first $Z$ matrix is constructed, $Z^{(1)}$ as the average of the corresponding elements of $X_{1}$ and $X_{2}$. Next, $X_{3}$ is rotated to fit $Z^{(1)}, Z^{(2)}$ is computed from $Z^{(1)}$ and $X_{3}$, etc., until $X_{N}$ is included in the preceding process. This, then, completes the initial cycle, yielding our best initial estimate of $Z$.

(b) Each of the $N X_{i}$ 's are then rotated to fit the current best estimate of $Z$ and a new estimate for $Z$ is computed from the average of the $X_{i}$ 's fitted to the current estimate of $Z$. A goodness of fit index is computed for each $X_{l}, g_{i}=r^{2}\left(X_{i}, Z\right)$ and then we compute

$$
h=\frac{1}{N} \sum_{i}\left(1-g_{i}\right)^{1 / 2},
$$


which is taken as an overall measure of fit for each new estimate of $Z$. We repeat process (b) until $h$ ceases to drop by more than some prespecified threshold value.

The above iterative algorithm corresponds to the one proposed by Kristof \& Wingersky [1971]. Berge [1977] has recently analyzed this procedure and found that a slightly modified version satisfies a somewhat stronger necessary condition for minimizing $L$. However, neither procedure generally satisfies the sufficient condition presented by Fischer \& Roppert [1965] and Berge [1977]. Moreover, it is not clear yet what constitutes the necessary and sufficient conditions for the present problem. Empirically, there has been some evidence as to the superiority of Berge's algorithm. If more results point in this direction, it may be desirable to implement the Berge procedure for PINDIS instead of the Kristof-Wingersky algorithm presently used.

Gower [1975], who allows one to input raw score data matrices as $X_{i}$ 's, provides an (optional) additional transformation besides rotation/reflection and translation, i.e., a central dilation scalar $k_{l}$ for each $X_{i}$. These $k_{i}$ 's are used to adjust for differences in the range of scores used by different individuals under the constraint

$$
\sum_{i} k_{i}^{2} \operatorname{tr}\left(X_{i} X_{i}^{\prime}\right)=\sum_{i} \operatorname{tr}\left(X_{i} X_{i}^{\prime}\right)
$$

Since in PINDIS every $\operatorname{tr}\left(X_{t} X_{i}^{\prime}\right)=1$, so that $Z$ represents a directly interpretable "average subject", whereas Gower makes only the weaker norming $\sum_{i} \operatorname{tr}\left(X_{i} X_{i}^{\prime}\right)=N$, such scalar multipliers would make no sense here. As one of our reviewers has pointed out, however, $X_{t}$-conditional scalar multipliers would still be useful as a fit index for the relationship of any $X_{i}$ and $Z$, but our $r^{2}\left(X_{i}, Z\right)$ is actually closely related to $k_{i}$ if employed in this respect: $k_{i}^{2} \operatorname{tr}\left(Z Z^{\prime}\right)^{1 / 2}=r^{2}\left(\tilde{X}_{i}, Z\right)$.

For the norm of $Z$ the following relationship holds:

$$
0 \leq \operatorname{tr}\left(Z Z^{\prime}\right)^{1 / 2} \leq 1,
$$

which follows from

$$
\sum_{t} \operatorname{tr}\left(X_{i} X_{i}^{\prime}\right)=N \operatorname{tr}\left(Z Z^{\prime}\right)+\sum_{i} \operatorname{tr}\left(E_{i} E_{i}^{\prime}\right)
$$

where the left-hand side represents the total sum of squares of the individual configurations, which is equal to $N$ due to the prior norming of every $X_{i}$. Thus, $\operatorname{tr}\left(Z Z^{\prime}\right)$ is equal to one if and only if all $X_{i}$ 's are perfectly explained by $Z$. In other words, the squared norm of $Z$ represents the communality of the $X_{i}$ 's, which is perfect if and only if all $X_{i}$ 's are related to each other by rotations/reflections and translations. The $n \times m$ matrix $E_{i}$ contains the unexplained residuals.

Having produced a target configuration, we now address the various transformations on both $Z$ and $X$ required for maximizing the fit between them.

\section{Similarity Transformations}

The problem at this initial stage of a PINDIS analysis is to fit each $X_{i}$ to a given $Z$ by rigid motions and central dilations. Although this has already been accomplished in the process of deriving $Z$ described in the previous section, we shall now present the involved issues in somewhat more detail. (It might also be noted that $Z$ may be an externally provided hypothesis configuration, in which case the $Z$-generating process would, of course, be bypassed.)

Restricting the set of transformations to similarity mappings will lead to sub-optimal solutions in the case that the $X_{i}$ 's resulted from a previous nonmetric scaling, since one would not use all of the indeterminacies in the individual configurations. Nevertheless, such an approach is consistent with the general practice of considering MDS solutions as 
essentially interval scaled for a sufficient number of points. Since the group of rotations/ reflections, translations, and central dilations exhausts the set of admissible and practically relevant transformations for nonmetric representations quickly as $n$ gets ever larger, it has essentially the same effect as the more general group of isotonic mappings.

A least-squares solution for the present problem has been provided by Schönemann \& Carroll [1970]. It minimizes the function

$$
F=\operatorname{tr}\left[\left(\tilde{X}_{l}-Z\right)\left(\tilde{X}_{l}-Z\right)^{\prime}\right],
$$

where

$$
\hat{X}_{i}=k_{i}\left(X_{i}-j u_{i}^{\prime}\right) R_{i},
$$

with $R_{\imath}, j$, and $u_{l}$ defined as in (6) and $k_{i}$ being a central dilation scalar.

Since we seek a simultaneous solution for the unknowns, (10) can be written as $\tilde{X}_{i}=k_{i} X_{i} R_{i}$ $-j u_{i}^{\prime}$ in this context. One obtains as solutions:

$$
R_{i}=A B^{\prime}
$$

where

$$
A \Delta B^{\prime}=X_{i}^{\prime} M Z=C,
$$

the Eckart-Young decomposition of $C$, and $M=I-j j^{\prime} / n$. The columns of $A$ are the eigenvectors of $C C^{\prime}$, those of $B$ are the eigenvectors of $C^{\prime} C$, both ordered according to the size of their common eigenvalues contained in the diagonal matrix $\Delta$ [Schönemann, 1966]. For $u_{i}$ :

$$
u_{i}=\frac{\left(Z-X_{t} R_{t}\right)^{\prime} j}{n}
$$

The effect of $M$ is to center both $X_{t}$ and $Z$ at the origin (which has already been accomplished in the process of calculating $Z$ ). Also, $u_{\iota}$ has been taken care of implicitly above, since $X_{i} R_{i}+j u_{i}^{\prime}=X_{i} R_{i}+\left(j j^{\prime} / n\right)\left(Z-X_{i} R_{i}\right)=\left(j j^{\prime} / n\right) Z+\left(I-j j^{\prime} / n\right) X_{i} R_{i}=\left(I-j j^{\prime} /\right.$ $n) X_{i} R_{i}=X_{i} R_{i}$. So the problem quite simply reduces to an orthogonal procrustean rotation [Schönemann, 1966], since the fit index does not require explicit computation of $k_{t}$.

The analysis at this (and at every subsequent) phase is concluded with the computation of the appropriate individual fit measure, which is here $r^{2}\left(X_{i}^{*}, Z\right)$.

\section{Dimension-weighting Transformations}

The problem at this stage is to predict each $X_{i}$ by a dimensionally weighted $Z$. In the more restrictive model we use a $Z$ with a fixed orientation analogous to INDSCAL's $G$ :

$$
\min =\operatorname{tr}\left[\left(X_{t}-Z S W_{i}\right)\left(\tilde{X}_{i}-Z S W_{i}\right)^{\prime}\right],
$$

where $S^{\prime} S=S S^{\prime}=I, W_{t}$ is diagonal, and $\tilde{X}_{i}$ is defined as in (10). First, if we call the function to be minimized in (14) $f$, one finds via $\partial f / \partial u_{l}=0$ :

$$
u_{i}=(1 / n)\left(X_{i}-Z S W_{l} R_{i}^{\prime}\right)^{\prime} j \text {. }
$$

Since both $X_{l}$ and $Z$ are column centered and since this property is not destroyed by $S, W_{i}$, and $R_{i}^{\prime}$, respectively, the translation vector must be equal to $(0, \cdots, 0)$. In (14) it is assumed, however, that $Z$ remains centered at the origin. We shall now show that $Z$ 's origin is sufficient for an optimal fit in the dimension-weighting case. Consider the following generalized loss function, where another translation vector, $t_{i}$, has been added:

$$
\begin{aligned}
& F=\operatorname{tr}\left(A A^{\prime}\right), \\
& A=\left(Z-j t^{\prime}\right) S W_{l}-\left(X_{l}-j u_{i}^{\prime}\right) R_{l} .
\end{aligned}
$$


Via partial differentiation one finds:

$$
\begin{aligned}
t_{i} & =\frac{1}{n}\left[Z-\left(X_{i}-j u_{i}^{\prime}\right) R_{i} S^{\prime} W_{i}^{-1}\right]^{\prime} j, \\
u_{i} & =\frac{1}{n}\left[X_{i}-\left(Z-j t_{i}^{\prime}\right) S W_{i} R_{i}^{\prime}\right]^{\prime} j .
\end{aligned}
$$

Inserting one solution into the other, one obtains $u_{i}=u_{i}$ and $t_{i}=t_{i}$, respectively, which proves the assertion. This result also shows that it is not necessary to center $Z$ at the origin for obtaining an optimal fit for dimension weighting, but it is definitely convenient computationally and is essential for a direct interpretation of the dimension weights.

To find $W_{i}$, we set $R_{i}=I=S$ for the moment. The optimal dimension weights are then given by

$$
\left[\operatorname{diag}\left(Z^{\prime} Z\right)\right]^{-1}\left[\operatorname{diag}\left(Z^{\prime} X_{i}\right)\right]=W_{i},
$$

i.e., they are the regression weights in $Z W_{i}+E_{i}=X_{i}$. We can normalize $W_{i}$ such that

$$
r^{2}\left(X_{i}, Z W_{i}\right)=\operatorname{tr}\left(W_{i}^{*} W_{i}^{* \prime}\right),
$$

by setting

$$
W_{i}^{*}=\left[\operatorname{diag}\left(Z^{\prime} Z\right)\right]^{1 / 2} W_{i}
$$

which follows from

$$
r^{2}\left(X_{i}, Z W_{t}\right)=\sum_{a}\left[\frac{\left(\sum_{p} x_{p a} z_{p a}\right)^{2}}{\sum_{p} z_{p a}^{2}}\right] \text {, }
$$

where the weights $w_{a a}=\sum_{p} x_{p a} z_{p a} / \sum_{p} z_{p a}^{2}$, given by (19), are used in simplifying the correlation equation.

We now formulate the following loss function for dimensions $a$ and $b(a \neq b)$ :

$$
K_{a b}=\frac{1}{\sum_{p} z_{p a}^{2}}\left(\sum_{p} x_{p a}^{*} z_{p a}\right)^{2}+\frac{1}{\sum_{p} z_{p b}^{2}}\left(\sum_{p} x_{p b}^{*} z_{p b}\right)^{2},
$$

where

$$
\begin{aligned}
& x_{p a}^{*}=x_{p a} \cos \alpha-x_{p b} \sin \alpha, \\
& x_{p b}^{*}=x_{p a} \sin \alpha+x_{p b} \cos \alpha .
\end{aligned}
$$

Since $K_{a b}$ represents the proportion of variance of $X_{i} R_{t}$ predicted by $Z W_{i}$ in the plane $a, b$, we want to maximize $K_{a b}$ for each pair of dimensions through an appropriate choice of the rotation angles, $\alpha$. For plane $a, b$ we obtain by differential calculus:

$$
\alpha=.5 \tan ^{-1}\left(\frac{2 A}{B}\right)
$$

where

and where

$$
\begin{aligned}
A & =\frac{C_{a a} C_{b a}}{V_{a}}-\frac{C_{b b} C_{a b}}{V_{b}}, \\
B & =\frac{C_{b a}^{2}-C_{a a}^{2}}{V_{a}}+\frac{C_{a b}^{2}-C_{b b}^{2}}{-V_{b}},
\end{aligned}
$$

$$
C_{i j}=\sum_{p} x_{p i} z_{p j} j V_{i}=\sum_{p} z_{p i}^{2}
$$


The rotation angle $\alpha$ will either maximize or minimize $K_{a b}$. The result depends upon the orientation of the initial $X_{i}$ relative to the maximizing/minimizing $X_{i}$. Because of (26), there are four special positions in a two space: two maxima and two minima. One can test if a rotation by $\alpha$ will maximize $K_{a b}$ by using the second derivative inequality:

$$
\frac{d^{2} K_{a b}}{d \alpha^{2}}=B \cos (2 \alpha)+2 A \sin (2 \alpha)<0
$$

and add $90^{\circ}$ to $\alpha$ if (29) does not hold, since it follows from (26) that maxima and minima alternate at $90^{\circ}$ intervals. In practice, $\alpha$ will rarely minimize $K_{a b}$ because the orientation of $X_{i}$ generated by the previous similarity transformations are almost always a very good initial estimate of the optimal $X_{i}$ for dimension weighting. However, in cases where the dimension weighting leads to very small weights for some coordinate dimensions of $Z$, i.e., where $Z$ is essentially collapsed to a lower dimensional space, the initial "similarity" orientation of $X_{l}$ might lead to a minimizing $\alpha$.

Rotations for higher dimensional spaces can be done pairwise for all ( $\left.\begin{array}{c}m \\ 2\end{array}\right)$ combinations of dimensions: rotate columns 1 and 2 of $X_{i}$ first, calculate their new values; rotate 1 and $3, \cdots$, rotate 1 and $m$; then continue with 2 and $3 ; 2$ and $4, \cdots, 2$ and $m$; until $m-1$ and $m$ complete one cycle. Repeat the cycles until some criterion of convergence is reached [cf., Harman, 1967]. For the reoriented $X_{i}$ 's optimal weights are then computed by (19), substituting $X_{l}$ by $X_{l} R_{t}$. The resulting solution is unique up to $180^{\circ}$ rotations in each plane for both $W_{i}$ and $R_{l}$.

The problem that remains to be solved in order to maximize the predictability of $X_{i}$ for the dimension-weighting model is to find that orientation of $Z$ with respect to some fixed coordinate system in which $Z$, when distorted along these axes, approximates $\tilde{X}_{l}$ as closely as possible. Apparently, every orientation of $Z$ is associated with a different family of producible configurations [cf., Lingoes \& Borg, 1977]. We shall first address the problem of finding the optimal rotation of $Z$ for each $X_{i}$ and then our attention will turn to finding the optimal orientation of $Z$ (fixed) over all $X_{i}$. The relevant loss function for the idiosyncratic case can be formulated as follows:

$$
\min =\operatorname{tr}\left[\left(Z S_{l} W_{i}-X_{i} R_{i}\right)\left(Z S_{i} W_{i}^{r}-X_{i} R_{i}\right)^{\prime}\right],
$$

where $S_{i}, R_{t}$, and $W_{t}^{r}=f\left(Z S_{t}, X_{t} R_{t}\right)$, which means that $W_{i}^{r} \neq W_{t}$ in general, are the unknowns. Stated for a two space, and using relationship (20) between normalized dimension weights and the variance explained in $X_{t}$ by $Z W_{i},(30)$ can be expressed as:

$$
\frac{1}{\sum_{p} z_{p a}^{* 2}}\left(\sum_{p} x_{p a}^{*} z_{p a}^{*}\right)^{2}+\frac{1}{\sum_{p} z_{p b}^{* 2}}\left(\sum_{p} x_{p b}^{*} z_{p b}^{*}\right)^{2}=\max
$$

where

$$
\begin{aligned}
& z_{p a}^{*}=z_{p a} \cos \beta-z_{p b} \sin \beta, \\
& z_{p b}^{*}=z_{p a} \sin \beta+z_{p b} \cos \beta,
\end{aligned}
$$

and $x_{p a}^{*}$ and $x_{p b}^{*}$ are defined by (24) and (25), respectively. Partial differentiation of (31) w.r.t. $\alpha$ and $\beta$ yields a simple solution for $\alpha$ (for some fixed $\beta$ ), corresponding to (26), but a very complicated expression for $\beta$ (holding $\alpha$ constant), which does not seem to be solvable in any simple way. Having by necessity to resort to a numerical approximation 
procedure, one first observes the following simplification: the optimal $Z$ must be attainable from the current $Z$ by a rotation of no more than $90^{\circ}$. This follows from a simple consideration: in a two space there are four possible positive directions for $Z$ which are related to each other by permutation transformations. In each of these orientations, $Z$ will explain the respective $\tilde{X}_{i}$ equally well. Thus, starting from some arbitrary orientation and rotating $Z$ over an interval of $90^{\circ}$ yields all possible communality values (i.e., $r^{2}$ 's) and, therefore, allows one to determine the optimal $Z$.

The search algorithm proceeds as follows: the original orientation of $Z$ is the one due to its generation in the Kristof-Wingersky process. Using this $Z$ as a starting point we compute the communality of $Z S_{i} W_{i}$ and $\tilde{X}_{i}$ with orthonormal $S_{i}$ rotating $Z$ in steps of $5^{\circ}$ over an interval $\pm 45^{\circ}$ in some plane $a, b$. The best $Z$ is found and a $\pm 5^{\circ}$ sector about this $Z$ is searched in $1^{\circ}$ intervals. The best $Z$ in this search process is then picked as the final $Z$ in a given plane, having an uncertainty level of $1^{\circ}$. This procedure is then iteratively repeated over all planes and each cycle until some criterion of convergence is satisfied. Although we have found empirically that the response surface is smooth and monotonically increasing to the maximum (which would allow one to use a more efficient algorithm), we could not show that the function will always possess these properties. By our search procedure, we obtain the optimal idiosyncratic $Z, Z_{i}^{r}$, for each $\tilde{X}_{i}$, which is unique up to axes permutations.

Determining an orientation for $Z$ (fixed) that is globally optimal would require the solution of (14) for $S$ over all $N X_{i}$ 's simultaneously, an apparently very difficult task in view of the dependency of the $W_{i}$ 's and the various transformations in $\tilde{X}_{i}$ on $S$. At this time, we cannot offer such a solution. However, extensive empirical analyses with artificial data have shown that the following procedure yields a very good approximation to the optimal $Z$ (fixed). In a two space, $Z S$ is taken as the arithmetic mean of the $Z_{i}^{r}$ s over all $N$ configurations. To show that $Z S$ calculated in this way is geometrically similar to each generating $Z$ one observes that the average of some matrix $A$ and another rotated matrix $A T\left(T T^{\prime}=T T=I\right)$ is similar to either one if and only if $.5(A+A T) .5(A+A T)^{\prime}=.5\left(A A^{\prime}\right)$ $+.5 A(T+T) A^{\prime}=k A A^{\prime}$, where $k$ is a scalar. For a two space one obtains $T+T^{\prime}=2 \cos$ $\alpha I$, but for a higher dimensional space $T+T^{\prime}$ is generally not a scalar matrix. This means that $\bar{Z}=1 / N \sum_{i} Z_{i}^{r}$ does not correspond to the rotated (and centrally dilated) average subject configuration for $m>2$. Expressed differently: an unknown distortion whose psychological meaning remains completely obscure would also affect $Z^{r}$ in this process. Furthermore, using $Z$ in lieu of the Kristof-Wingersky generated $Z$ for higher dimensional spaces would destroy the value of our basic reference index, since $r^{2}\left(\tilde{X}_{i}, \tilde{Z}\right)<r^{2}\left(\tilde{X}_{i}, Z\right)$, in general, whenever similarity is destroyed. Apparently, these features are undesirable. Thus, in order to preserve similarity we use $Z$ merely as a target to which $Z$ is orthogonally rotated as described above in the section on similarity transformations for $m>2$. Similarity should be preserved a fortiori whenever one has a hypothesis configuration, for obvious reasons.

\section{Vector-weighting Transformations}

On the next level of complexity, the loss function can be formulated as:

$$
\min =\operatorname{tr}\left\{\left[\tilde{X}_{i}-V_{i}\left(Z-j t_{i}^{\prime}\right)\right]\left[\tilde{X}_{i}-V_{i}\left(Z-j t_{i}^{\prime}\right)\right]^{\prime}\right\},
$$

where $\tilde{X}_{i}$ is defined as in (10) and $V_{i}$ is diagonal. Apparently, the attainable fit between $\tilde{X}_{i}$ and $V_{i}\left(Z-j t_{l}^{\prime}\right)$ in this vector-weighting model is independent of $Z$ 's orientation with respect to a given coordinate system, which simplifies the problem relative to the dimension-weighting case. However, (34) is more complicated in requiring the computation of two translation vectors, $u_{i}$ and $t_{i}$. The latter is necessary since the solutions for $u_{i}$ and $t_{i}$ do 
not lead to the tautology found in the dimension-weighting case, i.e.:

$$
\begin{aligned}
& u_{i}=\frac{1}{n}\left[X_{i}-V_{i}\left(Z-j t_{i}^{\prime}\right) R_{i}^{\prime}\right]^{\prime} j, \\
& t_{t}=\frac{1}{n}\left[Z-V_{i}^{-1}\left(X_{i}-j u_{i}^{\prime}\right) R_{i}\right]^{\prime} j .
\end{aligned}
$$

While the translation vectors were simple and, in particular, dependent in (17) and (18), this is not the case here. The complete solution of (34) requires, as a consequence, the determination of $t_{i}, u_{i}, R_{i}$, and $V_{i}$. Of these transformations only $R_{i}$ and $u_{i}$ are devoid of psychological significance. Translations and vector weightings for $Z$, on the other hand, are confounded and to some extent yield redundant information vis-à-vis one another, but may carry interpretative import.

Since there exist relatively direct solutions for the $R_{t}$ and $V_{i}$ as unknowns, we shall discuss their derivations first. Initially, one must determine the optimal reflection of $X_{i}$ relative to $V_{i} Z$. This step was, of course, irrelevant in the dimension-weighting case, since reflections could be handled there by the sign of the salience weight. We now make the following considerations: The criterion to be minimized is:

$$
D=\sum_{p} \sum_{a}\left(v_{p p} z_{p a}-x_{p a} \omega_{a}\right)^{2},
$$

where $\omega_{a}$ is either +1 or -1 . The vector weights $v_{p p}(p=1, \cdots, n)$ are determined from the regression equation $X_{i} \Omega_{i}=V_{i} Z+E_{i}$, where $\Omega_{i}$ is the $m \times m$ diagonal reflection matrix and $E_{i}$ are the residuals:

$$
\text { [diag } \left.\left(X_{i} \Omega_{i} Z^{\prime}\right)\right]\left[\operatorname{diag}\left(Z Z^{\prime}\right)\right]^{-1}=V_{i} .
$$

Inserting $v_{p p}=\left(\sum_{a} x_{p a} \omega_{a} z_{p a}\right) / \sum_{a} z_{p a}^{2}$ into (37) and simplifying the expression yields:

$$
D=-\sum_{p} \frac{\left[\sum_{a} x_{p a} \omega_{a} z_{p a}\right]^{2}}{\sum_{a} z_{p a}^{2}}-\sum_{p} \sum_{a} x_{p a}^{2} .
$$

Since the sum of squares of $X_{i}$ is unity due to norming, it follows that the first term in (39) has bounds zero to one. The task is then to maximize this term, which can be conceived of, analogous to the $W^{*}$ weights, as the sum of squares of normalized vector weights, $V^{*}$ :

$$
v_{p p}^{*}=v_{p p}\left(\sum_{a} z_{p a}^{2}\right)^{1 / 2}
$$

The sum of squares of these $v_{p p}^{*}$ represents the proportion of variance of $X_{i} \Omega_{i}$ accounted for by $V_{l} Z$. One observes immediately that $D$ is not differentiable with respect to the unknowns $\omega_{a}(a=1, \cdots, m)$. Consequently, we resort to a search algorithm in which each dimension is reflected in turn and the outcome is evaluated for any increase in the total sum of squared normalized vector weights. This single dimension approach, however, does not guarantee finding the best reflection of the $X_{l}$ under all conditions, but is, nonetheless, implemented for reasons that will be discussed in fuller detail after we deal with the problems of rotation and translation.

Having obtained an "optimally" reflected $X_{i}$, we proceed in a completely analogous manner to the dimension-weighting approach for rotation, i.e., in each plane we minimize 
(37), where $x_{p a} \omega_{a}=\hat{x}_{p a}$ is replaced by a rotated $\hat{x}_{p a}$. This leads to our plane-wise loss function of $D$ in (37):

$$
D_{a b}=\sum_{p}\left[\left(v_{p p} z_{p a}-x_{p a}^{*}\right)^{2}+\left(v_{p p} z_{p b}-x_{p b}^{*}\right)^{2}\right]
$$

where

$$
v_{p p}=\frac{\sum_{a} x_{p a}^{*} z_{p a}}{\sum_{a} z_{p a}^{2}},
$$

and $x_{p a}^{*}$ and $x_{p b}^{*}$ are as in (24) and (25), respectively, if one replaces $x_{p a}$ and $x_{p b}$ by $\hat{x}_{p a}$ and $\hat{x}_{p b}$. Inserting appropriately, differentiating the expression in (41) with respect to $\alpha$, and setting the derivative equal to zero yields:

$$
\alpha=.5 \tan ^{-1}\left(\frac{2 A}{B}\right)
$$

where

$$
\begin{aligned}
& A=\frac{\sum_{p}\left[z_{p a} z_{p b}\left(\hat{x}_{p a}^{2}-\hat{x}_{p b}^{2}\right)-\hat{x}_{p a} \hat{x}_{p b}\left(z_{p a}^{2}-z_{p b}^{2}\right)\right]}{z_{p a}^{2}+z_{p b}^{2}}, \\
& B=\frac{\sum_{p}\left[\left(\hat{x}_{p a}^{2}-\hat{x}_{p b}^{2}\right)\left(z_{p a}^{2}-z_{p b}^{2}\right)+4 \hat{x}_{p a} \hat{x}_{p b} z_{p a} z_{p b}\right]}{z_{p a}^{2}+z_{p b}^{2}} .
\end{aligned}
$$

As before, for dimension weighting, we test via the sign of the second derivative of (41) as to whether $\alpha$ maximizes or minimizes $D_{a b}$. If the former is true, $\alpha$ is incremented by $90^{\circ}$. With these plane-wise solutions we proceed iteratively until the cycles converge. Although we cannot offer a convergence proof for this process, abundant evidence from both artificial and empirical data strongly suggests convergence and the "recovery" of known a priori vector weights can be demonstrated.

The remaining problem at this stage of the analysis is to find the individual translation vectors $t_{i}$ and $u_{t}$. The problem is complicated by the fact that although one can express $t_{i}$ in terms of $u_{i}$, no simple analytic solutions could be derived for all the unknowns involved in the optimization of (37). Hence, we employed an ALS procedure which first solves for $V_{i}$ and $R_{i}$ with $t_{t}$ initially a null vector, then for $t_{i}$ [with $u_{i}$ substituted in (36) by (35)] in terms of the previous solutions, etc., until this process converges (necessarily). The fit values for the vector-weighting model with idiosyncratic translations on $Z$ (the unrestricted "perspective model", v.i.) are then computed on termination of the ALS procedure. These values are denoted by $r^{2}\left(\tilde{X}_{i}, V_{i}^{t} Z_{t}^{t}\right)$.

Now, in order to determine the overall optimal translation of $Z$ for the restricted perspective model, we proceed completely analogous to the dimension-weighting approach (where, it will be recalled, we determined from the average of the idiosyncratically rotated $Z$ 's, the $Z^{r}$ ). That is, by setting $t=1 / N \sum_{t} t_{t}$, the vector of average shift, we translate $Z$ to this new origin. By another ALS procedure, this time involving $Z^{t}=Z-j t^{\prime}$, one obtains the individual vector weights for the less general version of the perspective model. The fit values are now expressed as $r^{2}\left(\bar{X}_{i}, V_{i} Z^{t}\right)$, where $Z^{t}$ is fixed for all $N$ configurations. It should be clear that $V_{i} \neq V_{i}$, in general. Moreover, the full model will explain each $X_{i}$ at least as well as the fixed origin model, of course. 
As an alternative to shifting $Z$ by $t$, one may want to select some specific point as the origin of $Z$ (or leave the origin undisturbed in the case of some factor solution). In certain instances, e.g., in a radex structure, a meaningful origin is given and this is, of course, to be preferred to some blindly determined mathematical optimum. Thus, $Z$ may optionally be translated (or be left undisturbed) to a prespecified origin in a manner parallel to the option available in the dimension-weighting approach vis-à-vis the orientation of $Z$.

Some comments are in order regarding optimal reflections in vector weighting. It will be recalled that we stated that our reflection procedure for $X_{i}$ could be suboptimal under some conditions. We shall now take a closer look at this problem.

The problem is to reffect $X_{i}$ by $\Omega_{i}$ such that $\hat{X}_{i}$ will lead to a maximal fit if $\hat{X}_{i}$ is subsequently optimally transformed by a proper rotation $R_{i}$. Anticipating the proper rotation reduces the set of distinct reflection matrices to the relevant equivalence classes of $\Omega_{l}$ defined by all permutations of $m-1$ elements " 1 " and "-1". One observes that the rotation angle $\alpha$ in (43) is not identical over equivalence classes, however, since the signs in between the numerator terms of (44) and (45) would not be the same in all planes for different $\Omega$ 's. The latter observation implies that minimizing $D$ in (39) through an appropriate choice of the signs of each dimension in $X_{i}$ will not necessarily be optimal if one takes into account the possibility of later proper rotations. Examples illustrating this fact are readily constructed. Consider the three matrices below:

\begin{tabular}{rrrrrr}
\multicolumn{2}{r}{$Z$} & \multicolumn{2}{c}{$X$} & \multicolumn{2}{c}{$Y$} \\
-3 & 2 & & -6 & -1 & -6 \\
6 & 8 & -2 & -5 & 2 & -5 \\
1 & -7 & 3 & 4 & -3 & 4 \\
-5 & -4 & -7 & 8 & 7 & 8
\end{tabular}

Matrix $X$ is related to $Y$ by a reflection of the first dimension. The fit index $L=$ $\sum_{p}\left[\left(\sum_{a} x_{p a} z_{p a}\right)^{2} / \sum_{a} z_{p a}^{2}\right]$ has the value $L(Z, X)=57.07$. The analogous measure on $Y$ yields $L(Z, Y)=142.78$, which would suggest that either dimension of $X$ be reflected (which one is of no consequence). Computing the rotation angles we find $\alpha(X)=-16.26^{\circ}$ and $\alpha(Y)=-17.79^{\circ}$, but according to the second derivative test we must alter $\alpha(X)$ to $73.74^{\circ}$. We then obtain $L(Z, X R)=155.29$ and $L(Z, Y R)=152.15$, i.e., a relative reversal in the order of agreement so that we would have been better off not to have reflected $X$ at all.

To insure that (34) is minimized over all $\Omega$-equivalences we would have to test each and every one, a considerable undertaking for planar rotations if $m$ is of substantial size, i.e., for each $\Omega_{i}$ there would be

$$
\left(\begin{array}{c}
m \\
2
\end{array}\right)(m+1)
$$

rotations for each cycle. For $m=10$ one would need 495 planar rotations for one cycle and probably anywhere up to 50 cycles (on the average) would be required for convergence.

Although for the (not so uncommon) case of low dimensional spaces, the computational efforts would be much reduced, some other alternative is needed in general. Our present suboptimal algorithm is certainly inexpensive compared to the exhaustive requirements outlined above. In addition, it might be mentioned that extensive computer runs have demonstrated that reflections are in general not required and that when they are, the gain in fit is not of a magnitude that would tip the balance in favor of the vector model over that of others addressed by PINDIS, e.g., the dimensional salience model. The chief 
reason that reflections are not generally needed is that the dimension-weighting transformations place $X$ in an orientation that is not far from the optimal for that of vector weighting.

In our matrix formulations of the PINDIS hierarchy of transformations, v.i., we outline a possible ALS procedure for handling the vector-weighting case optimally in respect to both reflection and rotation. Before we delineate these matrix formulations for both dimension and vector weighting, we would like to make a few passing remarks on the uses and possible interpretations of the perspective model.

There are essentially two roles for the vector-weighting approach. The weight profiles may be indicative of more complicated relations among the subject configurations than is conveyed by a simple dimensional distortion. Additionally, the vector weights may also be related to a psychological model about inter-individual differences.

In the first case, where vector weighting is used as an index-providing procedure, one obtains, in particular, insight into the degree to which certain points in $Z$ are related to those in an individual's configuration in a way that cannot be handled by the affine transformations in the dimensional salience model. One may find that $Z$ explains an $X_{i}$ fairly well except for a few points that require substantial displacements. Such cases have been reported by Borg \& Lingoes [1976]: some individuals associated certain breakfast food items that were grouped by the average subject into some cluster $A$, say, with those in some other cluster, non- $A$. Except for those few points, however, they perceived the stimuli in practically the same way as the others. It may have been true though that all points required considerable shifting for all individuals and, at the same time, some typical patterns in the computed vector weights could be discerned over all subjects; this would then indicate that instead of computing $Z$ over apparently different groups, it might be useful to consider partitioning the sample first on the basis of the obtained vector weight profiles into more homogeneous groups. What one could aim at, for example, would be a partitioning such that the subjects in each subgroup were related to their common average by such simple transformations as a similarity transform or as dimensional weighting.

As in the dimensional salience model, the vector-weighting model is closely related to a psychological theory in a number of contexts. We choose to call this psychological model a perspective model for obvious reasons related to the geometry of the representation as it relates to visual perception. As an illustration, let us assume that one asks different individuals to compare various geographic locations with respect to the time they think it will take them to walk there from a given point. Let us further assume that the physical terrain is fairly hilly. Then one would expect that the subjects would radially shift the stimulus representations according to their respective perceived accessibilities. Physically very fit persons, for example, would probably use very little shifting as a function of the object's position on a hill or in a valley, whereas this would not be true for those who expect greater differences in required efforts. A number of psychological parallels to this physical example come readily to mind.

A relatively direct analogy exists for representations of sociometric data. An individual data matrix in this context may be as follows: row and column entries correspond to the same persons: $A, B, C, \cdots, Y$, where $A$ represents the experimental subject; the matrix elements stand for the similarity of each pair of persons as perceived by $A$. Scaling each such data matrix one obtains a spatial representation of the group structure for each individual $A, \cdots, Y$. One can now hypothesize that inter-individual differences are due to idiosyncratic origins (i.e., each subject is himself a point of perspective) and differential gradients due to liking, power, etc. One would thus assume that the individuals have basically the same perception of the group structure except for differences in the sense of the perspective model.

Another application of the perspective model is possible in the context of Guttman's 
radex theory [1954]. To illustrate this we consider a series of studies on "quality of life" [cf., for example, Levy \& Guttman, 1975], where item points in the SSA (smallest space analysis) space could be grouped into regions representing questions on satisfaction with one specific aspect of life. These regions were arranged as slices around an origin represented by the item "satisfaction with life in general". For such studies it was observed that different groups were different essentially only in shifting the points within each of these sectors [Levy, 1976; Borg, 1978]. Thus, the origin and also the order of the sectors remained practically the same for the groups and the point weighting was done in the sense of the perspective model. The radial shifting in this example is, therefore, related to differential perceptions of an item's centrality with respect to the issue "quality of life".

Although we have indicated some psychological interpretations for idiosyncratic origins and (non-negative) vector weights, there does not seem to be any convincing interpretation for negative vector weights in a model sense. This situation is similar, to some extent, to the dimensional salience case (for PINDIS, but not INDSCAL), where negative weights have a double meaning, i.e., they indicate both a differential importance and a necessary reflection of $Z$. The latter has the status of an index rather than that of a model parameter. For vector weights it seems most appropriate to treat the negative weight case similarly.

\section{Matrix Solutions for Dimension and Vector Weighting}

We shall now summarize the PINDIS model family in the more compact language of matrix algebra. In addition, we shall consider one rather obvious extension of the various weighting models used so far.

In general, the following loss function must be minimized in PINDIS:

where

$$
\operatorname{tr}\left(E E^{\prime}\right)=\min _{\left[R_{t}, S_{t}, W_{t}, V_{t}, K_{t}, t_{i}, u_{t}\right]}
$$

$$
E=V_{i}\left(Z-j t_{i}^{\prime}\right) S_{i} W_{i}-k_{i}\left(X_{i}-j u_{i}^{\prime}\right) R_{i} .
$$

The different approaches discussed so far were different in attempting a solution of (45) for proper subsets of the set of variables only. The various subsets are different with respect to the mappings on $Z$. The transformations on $X_{i}$ are always completely optimized (although this is strictly true for $R_{i}$ and $u_{i}$ only, the central dilation scalar $k_{i}$ will be implicitly determined by the fit index). It should be clear that $R_{i}, k_{i}$, and $u_{t}$ will generally be different for the similarity transform, the dimension weightings, and the vector weightings. In no instance should one attempt to interpret any of these mappings, of course. The possible exception of using $k_{i}$ as a fit index has already been discussed. Only the transformations on $Z$ are principally of substantive interest.

In the dimension-weighting case, we set $V_{l}=I$ and $S_{l}=S$. In the idiosyncratic orientation weighting, $V_{i}=I$ as before, but $W_{t}$ will now be different from the weight matrix obtained for the fixed orientation case. We have denoted the idiosyncratic orientation weight matrix by $W_{i}^{r}$ above to indicate the conditionality of the resulting weights on how $Z$ is rotated. Finally, in vector weighting, $W_{i}=I$ and rotations of $Z$ are irrelevant so that one can set $S_{t}=S$ arbitrarily. For the full perspective model, the idiosyncratic case, $Z$ is translated to $Z_{t}^{t}$ using $t_{t}$. For an overall-optimally positioned $Z, Z^{t}$, one uses $t$ as a shift vector.

For dimension weighting with idiosyncratic orientation we obtain the following solutions via partial differentiation of (46) under the respective linear constraints:

$$
u_{i}=\frac{\left(X_{i}-Z_{i}^{r} W_{i}^{r} R_{i}^{\prime}\right)^{\prime} j}{n},
$$




$$
\begin{gathered}
W_{i}^{r}=\left[\operatorname{diag}\left(Z_{i}^{r \prime} Z_{i}^{r}\right)\right]^{-1}\left[\operatorname{diag}\left(Z_{i}^{r^{\prime}} X_{i}^{u} R_{i}\right)\right], \\
\left(X_{i}^{u \prime} Z_{i}^{r} W_{i}^{r}\right) R_{t}^{\prime}=\text { symmetric, } \\
\left(W_{i}^{\prime \prime} Z^{\prime} X_{i}^{u} R_{i}\right) S_{l}^{\prime}=\text { symmetric },
\end{gathered}
$$

where $X_{i}^{u}=X_{i}-j u_{i}^{\prime}$. As has been shown above, a single translation vector is sufficient for optimization of (46) with $V_{i}=I$. Thus, $Z$ remains centered at the origin, i.e., $t_{i}=(0, \cdots$, $0)$. The solution (50) is due to minimization of (46) with $V_{i}=I$ and $t_{i}=(0, \cdots, 0)$ with respect to $R_{i}$ under the condition $R_{i}^{\prime} R_{i}=R_{i} R_{l}^{\prime}=I$. The latter constraint can be formulated as $\operatorname{tr}\left[L\left(R_{i}^{\prime} R_{i}-I\right)\right]=0$, where $L$ is a matrix of Lagrangian multipliers, which leads to the function $f=\operatorname{tr}\left(E E^{\prime}\right)+\operatorname{tr}\left[L\left(R_{i}^{\prime} R_{i}-I\right)\right]$. Solving $\partial f / \partial R_{l}=0$ yields

$$
n u_{i} u_{i}^{\prime}+X_{i}^{\prime} X_{i}+L=X_{i}^{u^{\prime}} Z S_{i} W_{i}^{r} R_{i}^{\prime},
$$

where the left-hand side is apparently a symmetric matrix. Now, setting $A=Z S_{i} W_{i}^{r}$ and $B=X_{i}^{u}$, it follows that $B^{\prime} A R_{i}^{\prime}=R_{i} A^{\prime} B$ or $B^{\prime} A=R_{i} A^{\prime} B R_{i}$ which leads to Schönemann's [1966] solution for the orthogonal procrustean rotation, i.e., $R_{i}=W V^{\prime}$ with $W$ and $V$ being appropriately oriented eigenvectors of $B^{\prime} A A^{\prime} B$ and $A^{\prime} B B^{\prime} A$, respectively.

There are four unknowns in the system of equations (48-(51), i.e., $u_{i}, R_{i}, S_{i}$, and $W_{i}$. First, of course, one observes that (48) leads to the null vector for $u_{i}$ since both $X_{i}$ and $Z$ are columnwise centered and since this property remains unaffected by the other transformations. Although each of the remaining equations can be solved for any given independent variables, there does not seem to exist any apparent direct solution for the whole set. It would be possible, however, to use an alternating method analogous to the one used in INDSCAL and ALSCAL, for example. If the space were higher than two-dimensional, this would have the advantage over the scalar approach that the rotation matrices would be computed directly rather than in the plane-wise iterative manner, which cannot be proved to converge necessarily.

For the vector-weighting approach, we obtain the following equations:

$$
\begin{gathered}
u_{i}=\frac{\left(X_{i}-V_{i}^{t} Z_{i}^{t} R_{i}^{\prime}\right)^{\prime} j}{n}, \\
t_{i}=\frac{\left[Z-\left(V_{i}^{t}\right)^{-1} X_{i}^{u} R_{i}\right]^{\prime} j}{n}, \\
\left(X_{i}^{u^{\prime}} V_{i}^{t} Z_{i}^{t}\right) R_{i}^{\prime}=\text { symmetric, } \\
V_{i}=\left[\operatorname{diag}\left(X_{i}^{u} R_{i} Z_{i}^{\prime \prime}\right)\right]\left[\operatorname{diag}\left(Z_{i}^{t} Z_{i}^{t \prime}\right)\right]^{-1} .
\end{gathered}
$$

For $R_{i}$ one observes that by using Schönemann's [1966] results no separate computation of a reflection matrix $\Omega_{i}$ is necessary. Again, the whole system is not directly solvable, but requires an iterative approach, such as outlined for dimension weighting above.

In evaluation of the entire matrix approach, one could say that it has clear advantages over the scalar solutions previously discussed whenever the transformational analyses are carried out in higher dimensional spaces. In the rather frequent case of two dimensions, however, we obtained some direct analytic results via scalar methods which have the additional advantage of being computationally faster than those involving matrix manipulations.

To conclude this section we consider an extension where (46) is solved for all transformations listed in the set of variables, i.e., $Z$ is now simultaneously weighted from the left and the right. A less complete form of such double weighting has been investigated previously by Lissitz et al. [1976], although in a different context. The solutions for this model are easily derived and could be solved (at considerable expense) via the alternating least-squares approach. (In fact, the PINDIS program presently contains a double- 
weighting analysis as an option.) The major difficulty with this model is, of course, that it is neither in any obvious way related to a psychological model such as "dimensional salience", for example, nor is it generally useful in an indexing sense because it is simply too complex. A rather exotic application where a restricted double-weighting solution proved useful can be found in Lingoes [1978]. Moreover, using double weighting in a purely formalistic sense, we found that it did not lead to appreciable fit improvements in practically all case that were investigated. Thus, we do not pursue this approach here any further.

\section{PINDIS Examples}

\section{Feger Political Party Attitudinal Data}

To illustrate PINDIS in a real application we use some data collected and previously analyzed by Feger [1974]. The study involved the six major political parties in West Germany: 1, a nationalistic party; 2 , a very conservative party; 3 , a moderately conservative party; 4 , the Liberals; 5 , the Social Democrats; and 6, the Communists. Fourteen subjects were asked to indicate which one of various combinations of political parties $(1=$ NPD, $2=\mathrm{CSU}, 3=\mathrm{CDU}, 4=\mathrm{FDP}, 5=\mathrm{SPD}, 6=\mathrm{DKP})$ and rating categories $(A=$ extremely far, $B=$ very far, $C=$ far, $D=$ close, $E=$ very close, $F=$ extremely close) were more descriptive of their attitudes. For example, the subject was presented with the two combinations $A-1$ and $D-4$. He then had to pick one of the two which he thought was a truer description of himself. Assume he chose $A-1$. $A-1$.would then be assigned a score of one, while $D-4$ would receive a score of zero. In the process of presenting all 630 possible pairs of pairs (using a randomized sequence, a screen projection of the stimuli, and a machine response recording system) a $6 \times 6$ dominance matrix was constructed, whose entries ranged between zero (dominates none) to thirty-five (dominates all) [cf., Feger, 1974, for further details]. These fourteen rectangular dominance matrices were then analyzed by SSAR-I [Lingoes, 1973], which assumes between-set information only (i.e., parties and categories) at the ordinal level and all elements are comparable. In every case an almost perfect representation was possible in a two-space, the poorest fit having a stress less than .009 (form 1) for Subject 8 . The $14 X_{i}$ 's of order $12 \times 2$ served as the input for a PINDIS analysis.

As a first step, PINDIS computed a $Z$, which is represented graphically in Figure 1 below. The average subject obviously arranges the parties on an approximate simplex (dashed line), where the nationalistic party (point 1 ) forms one terminus and the Communists (point 6), the other. The rating categories do not, surprisingly enough, form a simplex (solid line): the category "extremely close" is avoided by 12 of the 14 subjects and is positioned closer to the centroid of the parties. The squared norm of $Z$ was .75, which means that $Z$ accounted for $75 \%$ of the variance over all $X_{i}$ 's.

The first column of Table 2 lists the fit values for the similarity matching of the $X_{i}$ 's to $Z$. Apparently, most individuals had rather similar perceptions, which is inferred from their high communalities with the average subject $Z$. Individuals 1 and 9 , however, are much more poorly explained by $Z$ than are the other subjects. These differences are preserved even after dimension weighting (column 2 of Table 2). From a plot of Subject 1's perceptual space (see Figure 3), one can readily see that this subject does indeed have a configuration which cannot be satisfactorily related to $Z$ by either the similarity or dimension-weighting transformation. In general, we see from the second column of Table 2 that the dimension weights do not improve the fit values substantially for any subject, indicating that the computed weights should not be interpreted as differential dimensional saliences (the implicit unit weights of the similarity transform do almost as well). The information provided by a "subject space", i.e., a graphical representation of individual $w^{*}$ vectors in the stimulus space, as in INDSCAL, (Figure 2), should always be evaluated 


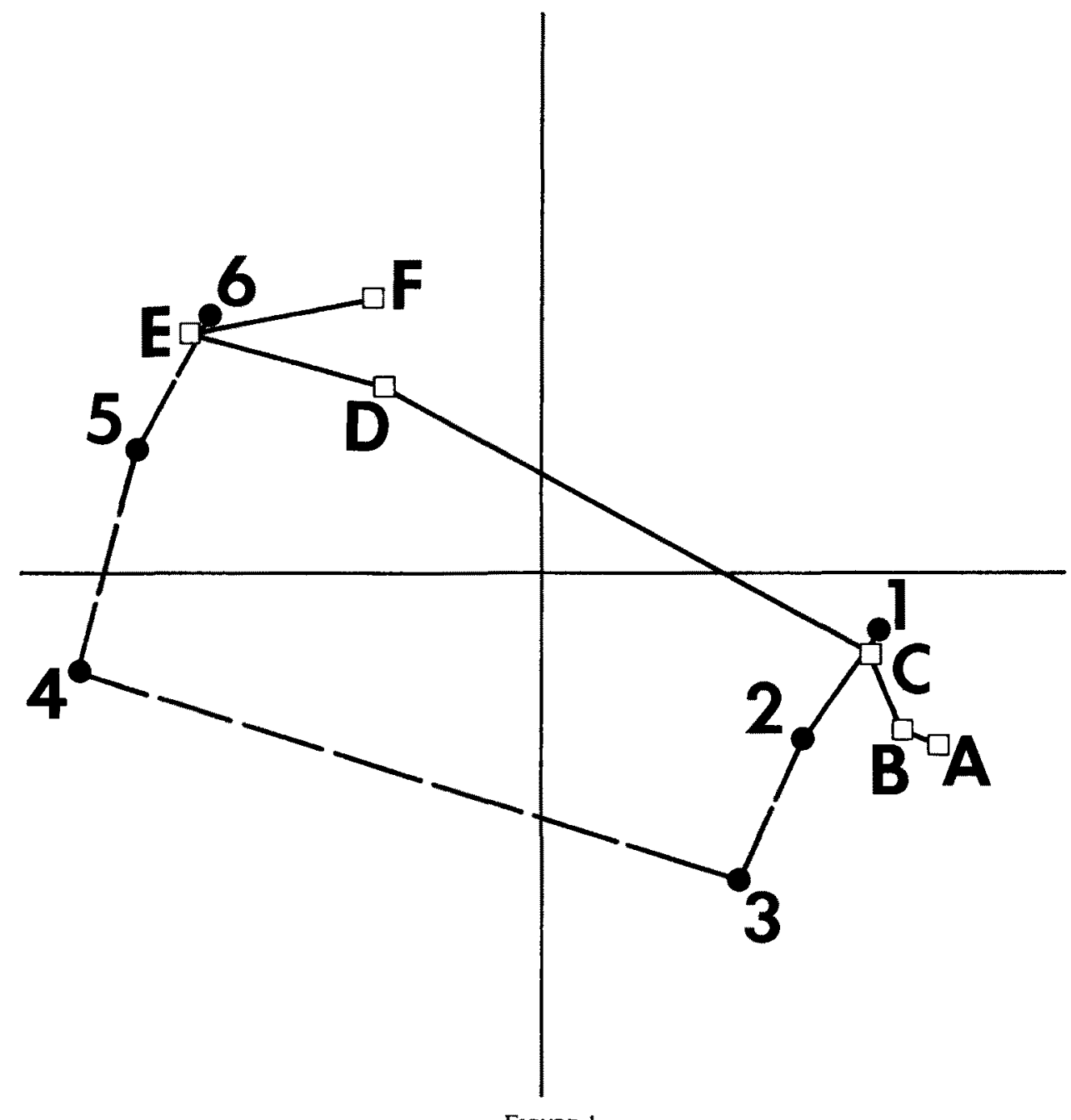

Figure 1

Centroid configuration for Feger data.

with care. The scatter around the origin can only be given a substantive meaning if the $X_{i}$ 's are substantially better explained under the dimension weighting transformation than by purely admissible, i.e., similarity mappings. The fact that we obtained comparable "communalities" from an INDSCAL analysis of the euclidean distances generated from the SSAR-I $X$ 's [Borg \& Lingoes, 1977] reinforces our conclusion. INDSCAL's $G$ was related to our $Z$ by a $180^{\circ}$ rotation and a simple dimension weighting to the extent of $r^{2}(G, Z)=$ 99.

Some useful additional information may be contained in column three of Table 2. Here, the communality between $\hat{X}_{i}$ and $Z_{l}^{r} W_{l}^{r}$, i.e., the individually optimally oriented $Z$, is represented. It is to be expected, of course, that there will be some dispersion of the $Z_{i}^{r}$ 's about $Z^{r}$. However, some $\tilde{X}_{i}$ 's may be substantially better explained by their respective $Z_{i}^{r}$ 's than by $Z^{r}$, which would indicate that these individuals are not likely to use the same spatial directions, or, in the terminology of IDIOSCAL, that they have idiosyncratic frames of reference. In our present example, this does not appear to be the case for any individual (only Subjects 7 and 9 reveal any tendency of this kind). If one found some instances of such idiosyncracy in ones data, it might be advisable to eliminate such 
TABLE 2

Fit Values for PINDIS Analysis of Feger Study

\begin{tabular}{|c|c|c|c|c|c|}
\hline \multicolumn{6}{|c|}{ FIT } \\
\hline Subject & $\mathbf{r}^{2}\left(\tilde{\mathbf{X}}_{\mathrm{I}}, \mathbf{Z}\right)$ & $r^{2}\left(\overline{\mathbf{X}}_{1}, Z^{r} W_{1}\right)$ & $r^{2}\left(\tilde{\mathbf{x}}_{i}, \mathbf{z r} \mathbf{w f}_{\mathbf{f}}\right)$ & $r^{2}\left(\tilde{\mathbf{X}}_{1}, \mathbf{v}_{\mathbf{i}} \mathbf{z}^{t}\right)$ & $\mathbf{r}^{2}\left(\tilde{\mathbf{x}}_{1}, V_{i}^{(} \mathbf{Z}_{i}^{t}\right)$ \\
\hline 1 & .155 & .167 & .168 & .674 & .885 \\
\hline 2 & .801 & .817 & .819 & .887 & .998 \\
\hline 3 & .942 & .948 & .948 & .986 & .998 \\
\hline 4 & .854 & .862 & .862 & .939 & .996 \\
\hline 5 & .780 & .785 & .785 & .942 & .994 \\
\hline 6 & .780 & .794 & .794 & .910 & .962 \\
\hline 7 & .877 & .881 & .915 & .965 & .950 \\
\hline 8 & .794 & .795 & .796 & .943 & .981 \\
\hline 9 & .584 & .586 & .643 & .885 & .942 \\
\hline 10 & .842 & .844 & .844 & .947 & .998 \\
\hline 11 & .945 & .945 & .945 & .986 & .998 \\
\hline 12 & .791 & .793 & .798 & .949 & .926 \\
\hline 13 & .838 & .849 & .849 & .984 & .975 \\
\hline 14 & .844 & .850 & .850 & .960 & .993 \\
\hline$r^{2}$ & .773 & .778 & .786 & .926 & .971 \\
\hline
\end{tabular}

individuals from the analysis and then re-analyze the data. In this manner, the sample could be partitioned into more homogeneous subgroups.

Proceeding with the vector-weighting transformation at the next level of complexity, we obtained the fit values given in the fourth column of Table 2, Overall, it is obvious that

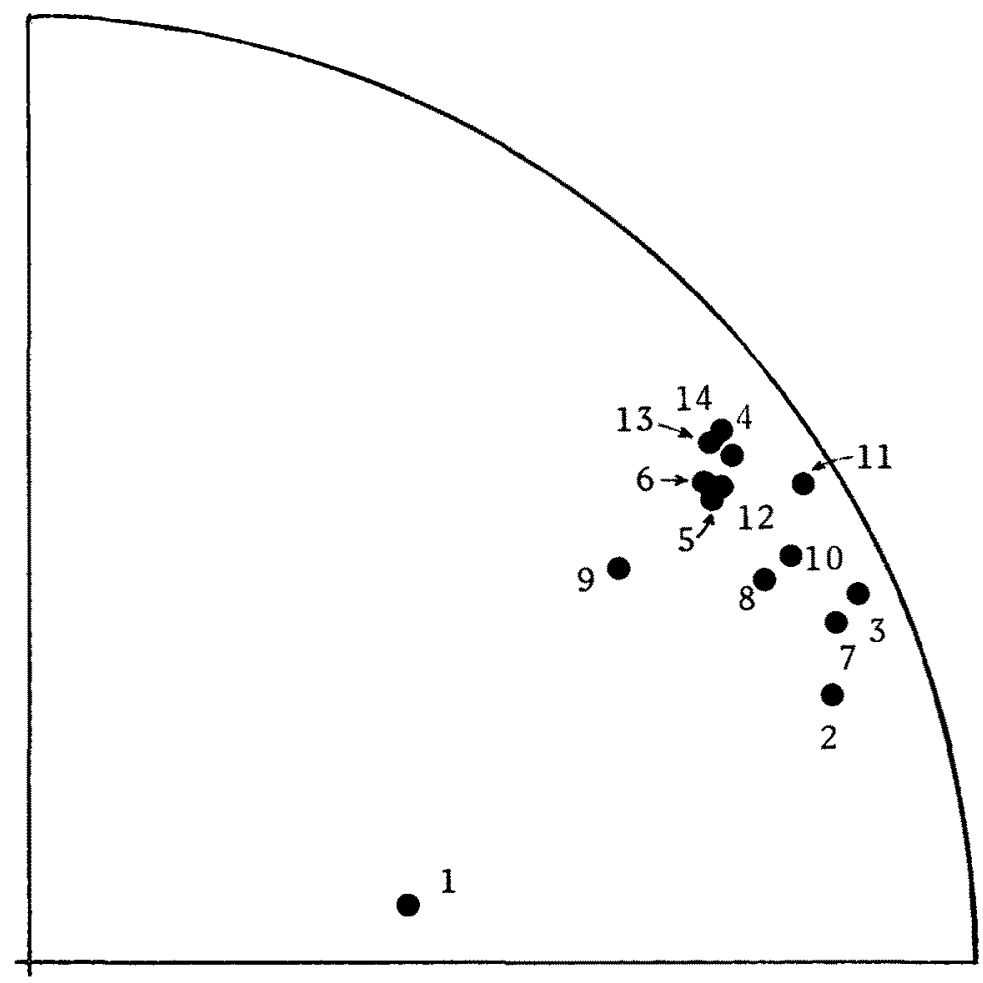

FIGURE 2

Subject space for Feger data. 
this transformation accomplished more than that of dimension weighting: The total communality improvement is $15 \%$ as compared to practically $0 \%$, respectively, over the similarity transform. This increment has to be evaluated, however, against the considerably increased number of free parameters in vector weighting [ 12 vs. 0 (similarity) and 12 vs. 2 (dimension weighting)]. Nevertheless, it is clear that, for example, for Individual 1 the fit improvement is quite impressive. Taking a closer look at this individual, it is interesting to note that practically all of his optimal vector weights are quite different among each other and they depart from +1 . Apparently, $Z$ explains $X_{1}$ sufficiently well only under independent (and substantial) movements of the points. Quite clearly, these point displacements show that his perceptual space relative to the one of the average subject is "scrambled". Indeed, a plot (see Figure 3 ) of his space shows that he folds what others perceive as a left-right simplex of political parties (Figure 1) at his most preferred party, which obviously generates a radically different perceptual space.

It may be noted that Individual 1's attitude structure showed remarkable stability over eight replications taken over eight weeks of testing [Feger, 1975; Borg \& Lingoes, 1977]. In other words, his discrepancy from the average subject cannot be explained by error alone. In conclusion, it becomes apparent that the vector weights can serve as yet another index of group homogeneity and, consequently, as a basis for evaluating the representivity of the average subject space in the dimensional salience model. This latter point simply means that $Z$ may be a confounding of essentially qualitatively different individual structures, or, in other words, the average subject may not represent any individual sufficiently well. If such is the case, then the dimensional salience model does not make much sense, of course.

Allowing for individual origins of the average subject configuration, one obtains the fit values found in the fifth column of Table 2 . On the average, a further fit improvement of some $5 \%$ over the previous transformation has been effected. However, the communality

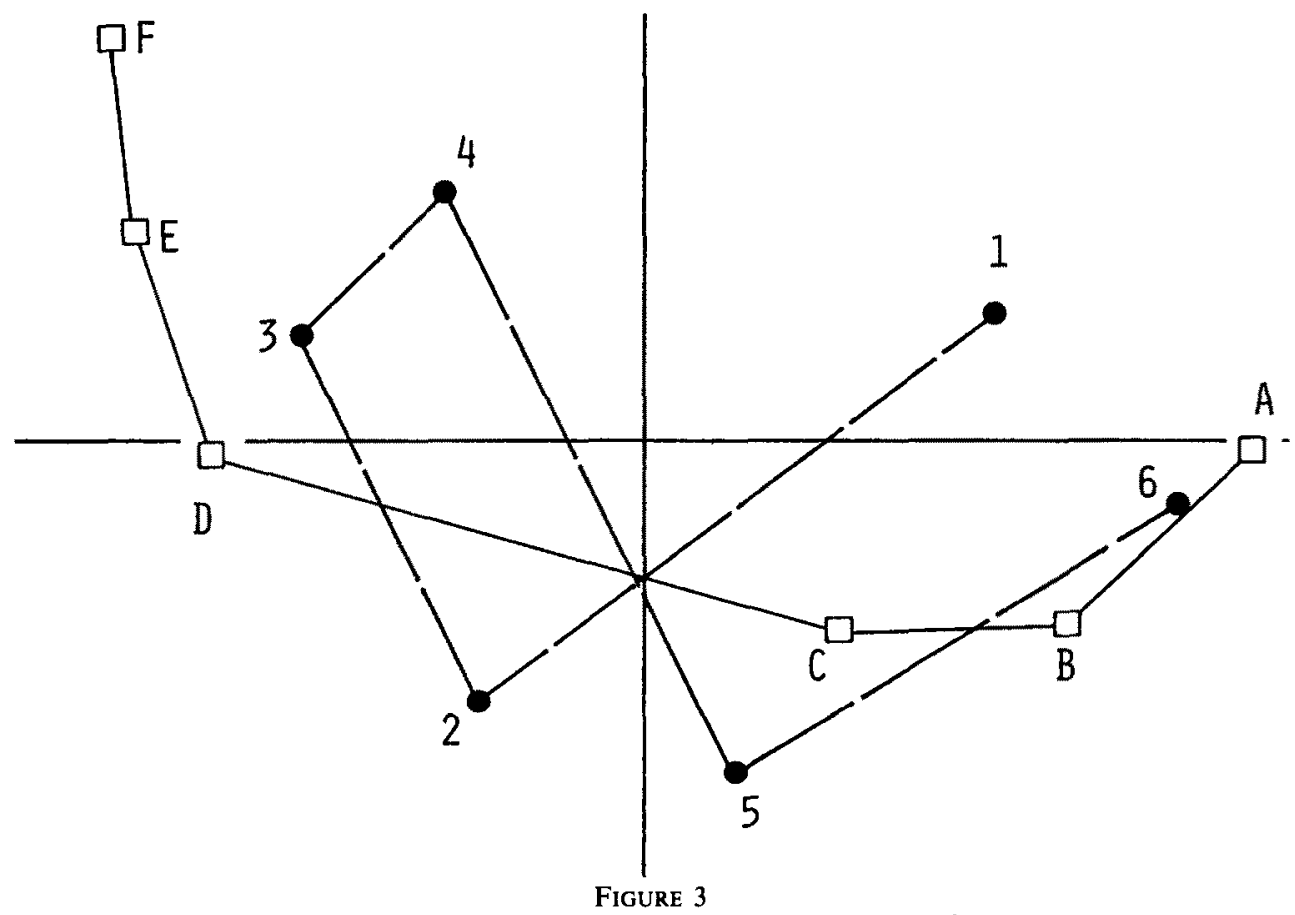

SSAR-I configuration for Individual 1 in Feger study. 
increments are not uniformly distributed over subjects. Individuals 7, 12, and 13 even drop slightly in their communalities, which appears to be a function of the flatness of their response surfaces and the termination criterion in the ALS procedure. On the other hand, one notes a substantial fit improvement for Subject 1 of over $20 \%$, indicating again that this individual is quite different from the remaining subjects. (Indeed, from other sources of information [Feger, Note 8] we know that Subject 1 is about forty years of age and has a conservative attitude in political matters, whereas the remaining thirteen subjects are psychology students with pronounced leftist views.) For the idiosyncratic origins, one observes considerable variance among the individuals (see Figure 4). However, taking into account the fact that appreciable fit improvement was obtained for two subjects only (1 and 2), it seems questionable to interpret the noted scatter as indicative of real interindividual differences for all subjects, particularly when one takes into account the relatively high fit values for the fixed origin version of the vector-weighting model. Under other conditions, of course, one could use the origin space in a manner similar to the subject (dimension weight) space as a basis for partitioning. For the present results, however, it seems reasonable to contrast the origin of Subject 1 with the average of the remaining individuals. Since no substantive perspective model was assumed to account for these data, only indexing information is herewith provided.

\section{Helm Color Data}

In this example we use data collected by Helm and analyzed by him [Note $5 ; 1964$ ] via a ratio scale MDS technique, by Helm \& Tucker [1962] via a points of view method, by Carroll \& Chang (as reported by Wish \& Carroll, 1974) via INDSCAL, and by MacCallum [1974] comparing INDSCAL with Tucker's three-mode scaling.

Helm [Note 5] asked subjects to judge the similarity of ten colors which differed in hue by arranging triples of color tiles such that the distances among the tiles were to be set proportional to their perceived similarities. The matrix of distances derived from these triadic judgements was assumed to be determined up to a scalar multiplier. Thus, the matrix of scalar products for each subject was diagonalized without prior transformations. There were ten color normal subjects plus one replication (normals) and four red-green deficient individuals (as determined by a standard discrimination test) plus one replication (deficients). The eigenvalues for the normals and mildly color deficient subjects indicated an underlying two space, whereas the two highly deficient subjects yielded but a single dimension essentially. Negative eigenvalues were obtained in all cases, but were explained by Helm [Note 5] and Helm \& Tucker [1962] to be the result of systematic estimation error.

The first two components of loadings from the normals were used as the PINDIS input. The resulting $Z$ is represented in Figure 5. The average subject configuration shows the expected color circle. The vertical axis corresponds directly to a red-green dimension relative to which we should anticipate finding the essential differences among color normals and deficients.

The five color deficients' two dimensional spaces from Helm's analysis were then related to this $Z$ by using the PINDIS option for fixing the target $(Z)$ and expressing all transformations in terms of the given $Z$ for each $X_{i}$. The fit values for all sixteen data sets are given in Table 3. Note that there is no idiosyncratic rotation or translation for the deficients, since $Z$ was fixed in the orientation and origin optimal for explaining the normals under the dimensional salience and perspective models, respectively.

Table 3 shows that $Z$ explains the $X_{i}$ 's of the normals extremely well without any differential weighting. This is reflected in Figure 6 by the fact that all the respective weight vectors (solid points) in the subject space are (a) closely clustered around the bisector and (b) lie almost at the unit circle, which expresses perfect communality. Consequently, 

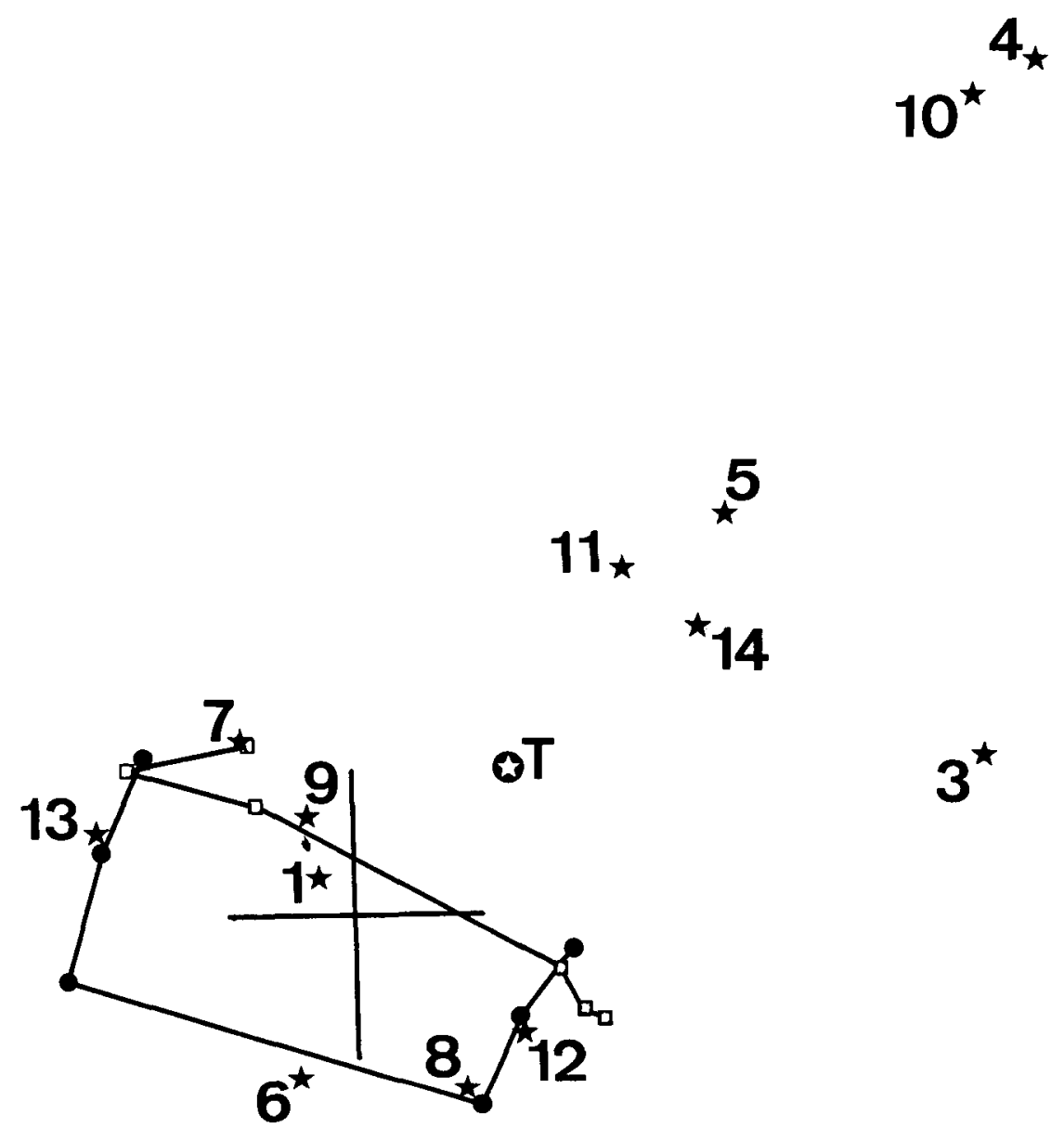

$\star^{2}$

Idiosyncratic origins (stars) for 14 subjects of Feger study. $\mathrm{T}=$ overall optimal origin.

neither idiosyncratic orientations of $Z$ nor vector weightings are of any relevance here. It should be mentioned that close clustering of subject points indicates highly similar private perceptual spaces, but that the points may scatter considerably even if very little additional variance, relative to the unit weighting implied by the similarity transform, is explained by 


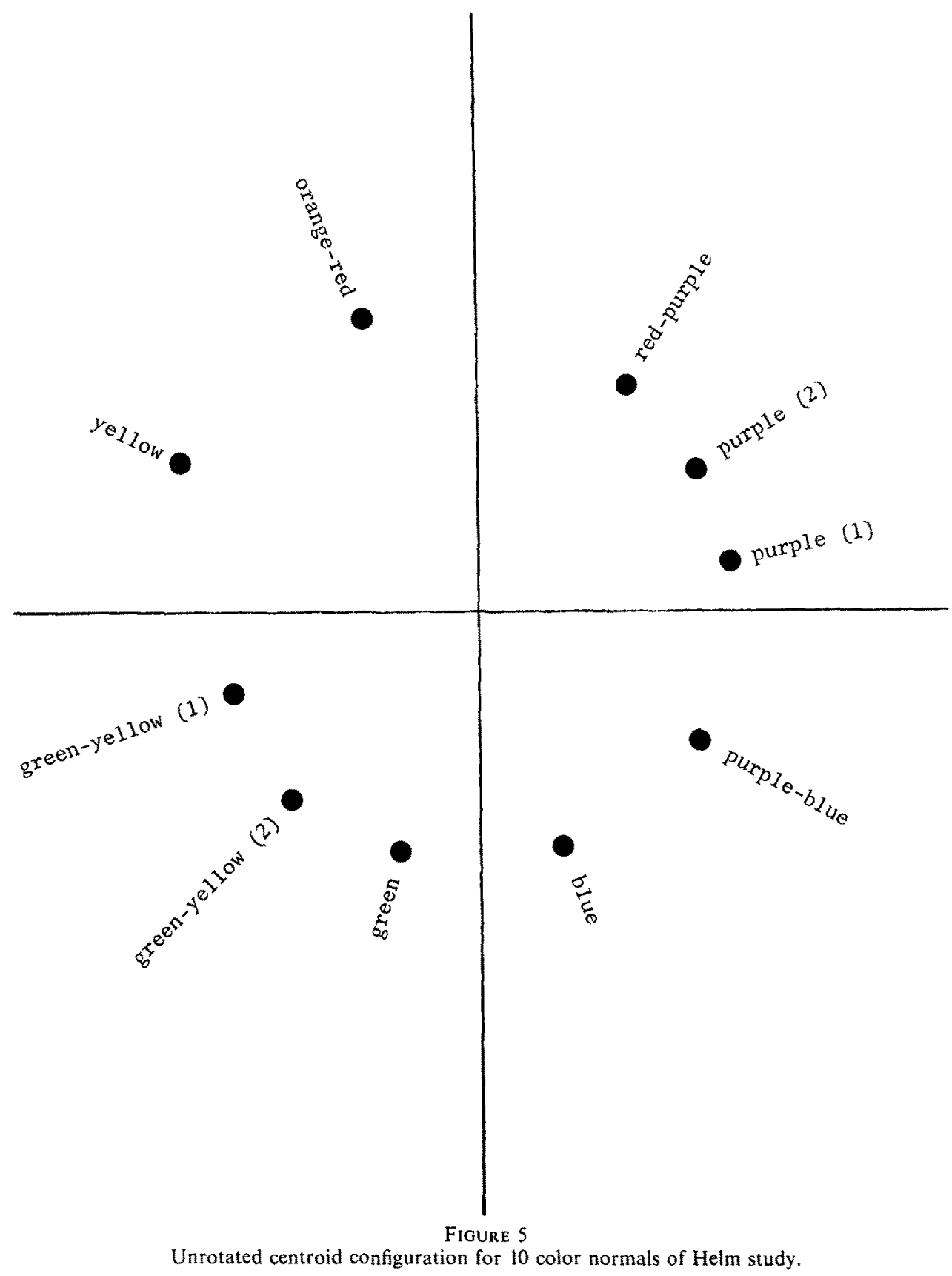

differential dimension weights [Borg \& Lingoes, Note 2]. This scatter should, therefore, only be interpreted if $r^{2}\left(X_{i}, Z\right) \ll r^{2}\left(X_{i}, Z^{r} W_{i}\right)$.

For the color deficients, Table 3 reveals that dimension weighting improves the fit on the average by some $15 \%$ and for the two most deficient individuals even higher. In the subject space one can see that the weight vectors for the deficients (stars) are clearly separated from the normals. Furthermore, the salience of the red-green dimension is, in all cases, lower than unity. The estimated private perceptual spaces would thus be ellipses rather than circles as for the normals. For the extremely deficient subjects these ellipses are 
TABLE 3

Fit Values for PINDIS Analysis of Helm Color Data

\begin{tabular}{|c|c|c|c|c|c|}
\hline \multirow{2}{*}{ Subject } & \multicolumn{3}{|c|}{ FIT } & \multirow[b]{2}{*}{$\mathbf{r}^{2}\left(\tilde{\mathbf{X}}_{1}, \mathbf{V}_{1} \mathbf{Z}^{\mathrm{t}}\right)$} & \multirow[b]{2}{*}{$\mathbf{r}^{2}\left(\tilde{\mathbf{X}}_{\mathrm{i}}, \mathbf{V}_{1}^{\mathrm{t}} \mathbf{Z}_{\mathrm{i}}^{\mathrm{t}}\right)$} \\
\hline & $\mathbf{r}^{2}(\hat{\mathbf{X}}, \mathbf{Z})$ & $\mathbf{r}^{2}\left(\overline{\mathbf{X}}_{1}, Z^{r} \mathbf{W}_{1}\right)$ & $\tilde{z}^{2}\left(\tilde{\mathbf{X}}_{i}, Z_{1}^{r} \mathbf{W}_{i}^{r}\right)$ & & \\
\hline \multirow{12}{*}{ 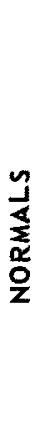 } & .978 & .983 & .983 & .995 & .998 \\
\hline & .978 & .981 & .987 & .998 & .998 \\
\hline & .990 & .991 & .992 & .997 & .999 \\
\hline & .989 & .990 & .991 & .994 & .998 \\
\hline & .991 & .991 & .991 & .996 & .999 \\
\hline & .969 & .970 & .971 & .985 & .998 \\
\hline & .983 & .883 & .988 & .993 & .997 \\
\hline & .974 & .974 & .975 & .984 & .997 \\
\hline & .982 & .983 & .983 & .989 & 1.000 \\
\hline & .983 & .991 & .992 & .992 & 1.000 \\
\hline & .956 & .965 & .967 & .982 & .995 \\
\hline & .979 & .982 & .983 & .991 & .998 \\
\hline \multirow{6}{*}{$\begin{array}{l}\tilde{L} \\
\mathbf{z} \\
\underline{u} \\
\frac{u}{u} \\
u \\
\Delta\end{array}$} & .891 & .920 & $-*$ & .966 & $-\dagger$ \\
\hline & .911 & .949 & - & .944 & - \\
\hline & .437 & .823 & $=$ & .772 & $\cdot$ \\
\hline & .943 & .968 & - & .977 & - \\
\hline & .496 & .754 & - & .776 & - \\
\hline & .736 & .883 & - & .887 & - \\
\hline
\end{tabular}

* Orientation fixed to optimal solution of normals,

$\dagger$ Origin fixed to optimal solution of normals.

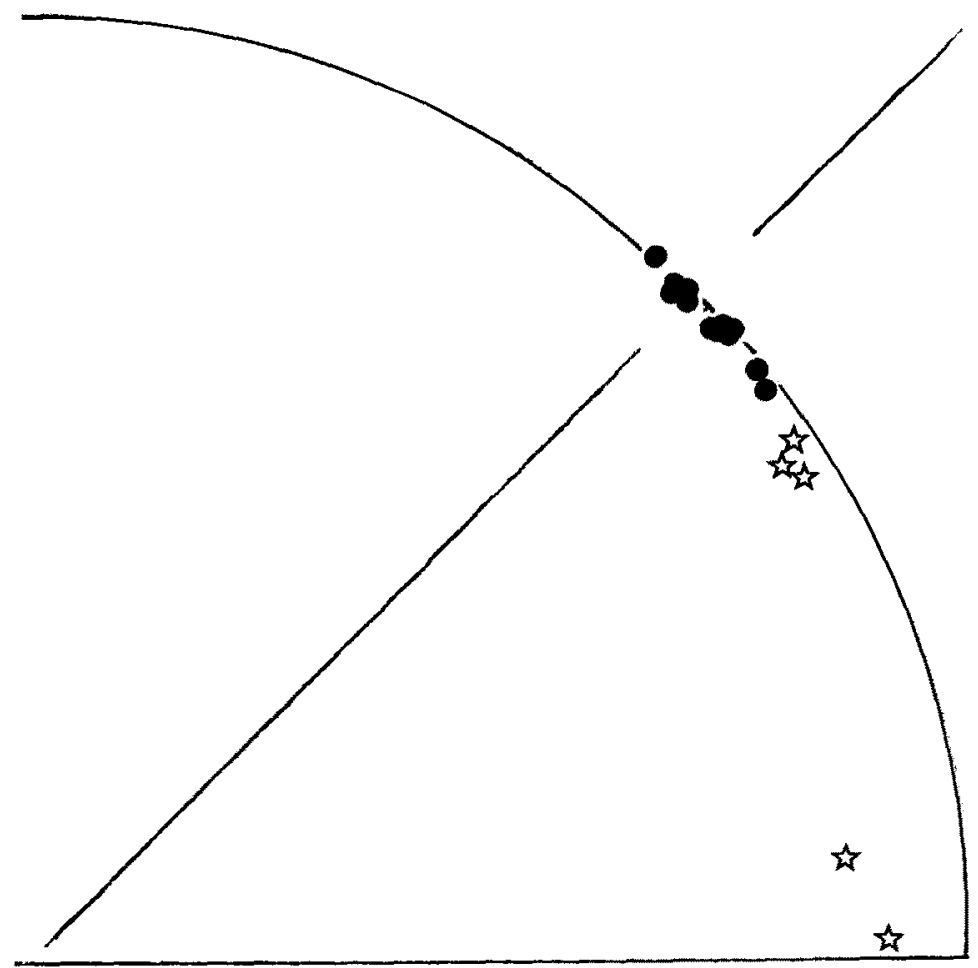

Figure 6

Subject space for Helm data. Dots $=$ normals, and stars $=$ deficients. 
almost collapsed onto a line, i.e., these subjects differentiate the colors only on a green/ yellow-purple continuum.

Table 3 further shows that the vector weightings do not indicate peculiarities in the perceptual space of any individual, since such weightings improve the fit either only by a negligible amount or lead to a substantial decrement as compared to dimension weighting. This observation is even more relevant if it is evaluated against the fact that eight (ten) additional free parameters are used here. The results, therefore, validate the expectations in all respects.

If we had no prior knowledge about the deficiency of some subjects, the $Z$ generated from all sixteen data sets would, of course, reflect the low salience of the red-green dimension for five individuals: $Z$ is then flattened to an ellipse along the red-green axis. The associated subject space does, however, still allow one to distinguish between the different subgroups, although the mildly deficients are only extremes of the group of normals (as can be seen from Figure 6). Note that in an INDSCAL analysis, the group space would not change its shape in the same manner, since it is normed to unit length on all dimensions; only the INDSCAL subject space would then reflect the fact that the average subject configuration is not circular.

Another interesting phenomenon can be observed if one analyzes the complete set of the $X_{i}$ 's made up of the first three and four dimensions based on Helm's solutions, respectively, rather than those corresponding to the first two dimensions only. The resulting fit values will then be poorer under all transformations (Table 4) except that of vector weighting. From this fact one has to conclude that the common perceptual structure is two rather than three or four dimensional. The additional dimensions constitute non-common variance essentially and may thus be regarded as error in this context. Of course, since neither one of the additional dimensions accounts for much variance relative to the first two, their effect on the total communality is rather small, i.e., they change the ratio of explainable to error variance only slightly in the direction of error variance.

We observe, however, that the communalities do not drop monotonically from two to four dimensions in all instances: for both dimension weighting transformations there is no such decrement. Taking a closer look at the individuals, one finds that the color deficients are explained slightly better by increasing dimensionality, whereas just the opposite is true for the normals. Thus, while added dimensions represent error for the normal subjects, this seems not to be the case for the deficients (an observation already made by Helm, Note 5).

One might pursue this issue of dimensionality further in the present case by analyzing

TABLE 4

PINDIS Average Common Variance

(by dimensionality) for Helm Data

\begin{tabular}{|c|c|c|c|}
\hline \multirow[b]{2}{*}{$\mathbf{r}$} & \multicolumn{3}{|c|}{ DIMENSIONALITY } \\
\hline & 2-D & 3-D & 4D \\
\hline$\tilde{\mathbf{x}}_{1}, \mathbf{z}$ & .91 & .88 & .88 \\
\hline $\mathbf{X}_{1}, \mathbf{Z}^{s} \mathbf{w}_{\mathrm{i}}$ & .95 & .92 & .92 \\
\hline$\tilde{\mathbf{x}}_{1}, \mathbf{z}_{1}^{r} \mathrm{~W}_{1}$ & .95 & .92 & .93 \\
\hline$\tilde{\mathbf{x}}_{1}, \mathbf{v}_{1} \mathbf{z}^{t}$ & .97 & .99 & .96 \\
\hline$\tilde{\mathbf{x}}_{\mathrm{i}}, \mathbf{v}_{\mathrm{i}}^{\mathrm{t}} \mathbf{z}_{\mathrm{i}}^{\mathrm{t}}$ & .99 & 1.00 & .99 \\
\hline
\end{tabular}


normals and deficients separately, which would be particularly interesting if a hypothesis about such a difference in the dimensionality of the perceptual spaces of normals and color deficients was to be given. In the absence of having some theoretical basis for deciding upon the dimensionality of the $X_{i}$ 's, one could to some extent use the observations made here, i.e., one could analyze $X_{i}$ 's in different dimensionalities and then determine where the maximal average fit occured. One should remember, however, that proceeding in this fashion does not guarantee optimal predictability within each meaningful subgroup. In general, at this stage of knowledge, we would hesitate in making any strong recommendations as to how one could determine the dimensionality of the $X_{i}$ 's on purely formal grounds. Clearly, further evidence is needed and substantive considerations play an important role here.

\section{Discussion}

In our second illustration, where we reanalyzed Helm's color data, PINDIS was applied in a confirmatory way. The orientation and origin of the average subject configuration obtained in the analysis of the color normals was such that it corresponded to the substantive theory for the differences among the individuals. Thus, exactly this average subject space was used as a not-to-be-changed hypothesis configuration in the subsequent scaling of the color deficient individuals. It might have been necessary, however, to rotate the centroid configuration to a meaningful orientation first before weights related to a substantive theory of spatial directions could be derived. Proceeding in this manner, one would generally not be able to predict the same amount of variance, of course, since $Z^{r}$ 's orientation is unique (mathematically) in the dimensional salience model. In some empirical tests where $Z$ was rotated over a $180^{\circ}$ interval, we observed the rather interesting behavior, however, that the decrement in predicted variance for non-optimal orientations relative to the optimal $Z$ was generally quite small. This finding requires further investigation, needless to add, but even at this stage of knowledge, we do not think that there can be much conflict for the researcher as to whether $Z$ should be "optimally" or "substantively" oriented.

In other applications [Borg, 1977] we have also observed that the similarity transformations do generally, by far, the most important job in predicting an individual from the average subject, although we have also found some instances where (fixed origin) vector weighting has increased the proportion of explained variance by some $65 \%$ [Borg \& Lingoes, Note 2]. It would be interesting to know in this context how well various random configurations could be explained by their centroids, of course, since we presently lack a statistical theory for our fit measures. Our choice of examples was in part dictated by the appropriateness of the two vis-à-vis the two models discussed, i.e., the dimension salience model (Helm's color data) and the perspective model (Feger's political attitude data), but it should be noted that over a wide range of data we have found the perspective model explains individual differences better than does the dimension salience model (which generally does little better than the unit weighting of the similarity transform). The difficulty in evaluating this finding in the absence of a statistical theory, however, leads us to suggest a certain amount of pragmatism in exploring both as a matter of routine.

As a final remark, we note that PINDIS provides normed scalars for each $X_{i}$ so that unconditional weights for both dimensions and vectors can be easily computed for correlational and group comparison purposes [MacCallum, 1977].

\section{Appendix: Definition of Symbols}

\section{Scalars and Miscellaneous}

$\begin{array}{ll}a, b & \text { dimension subscripts }(a, b=1,2, \cdots, m \text { dimensions }) \\ i & \text { configuration super/subscript }(i=1,2, \cdots, N \text {.configurations })\end{array}$




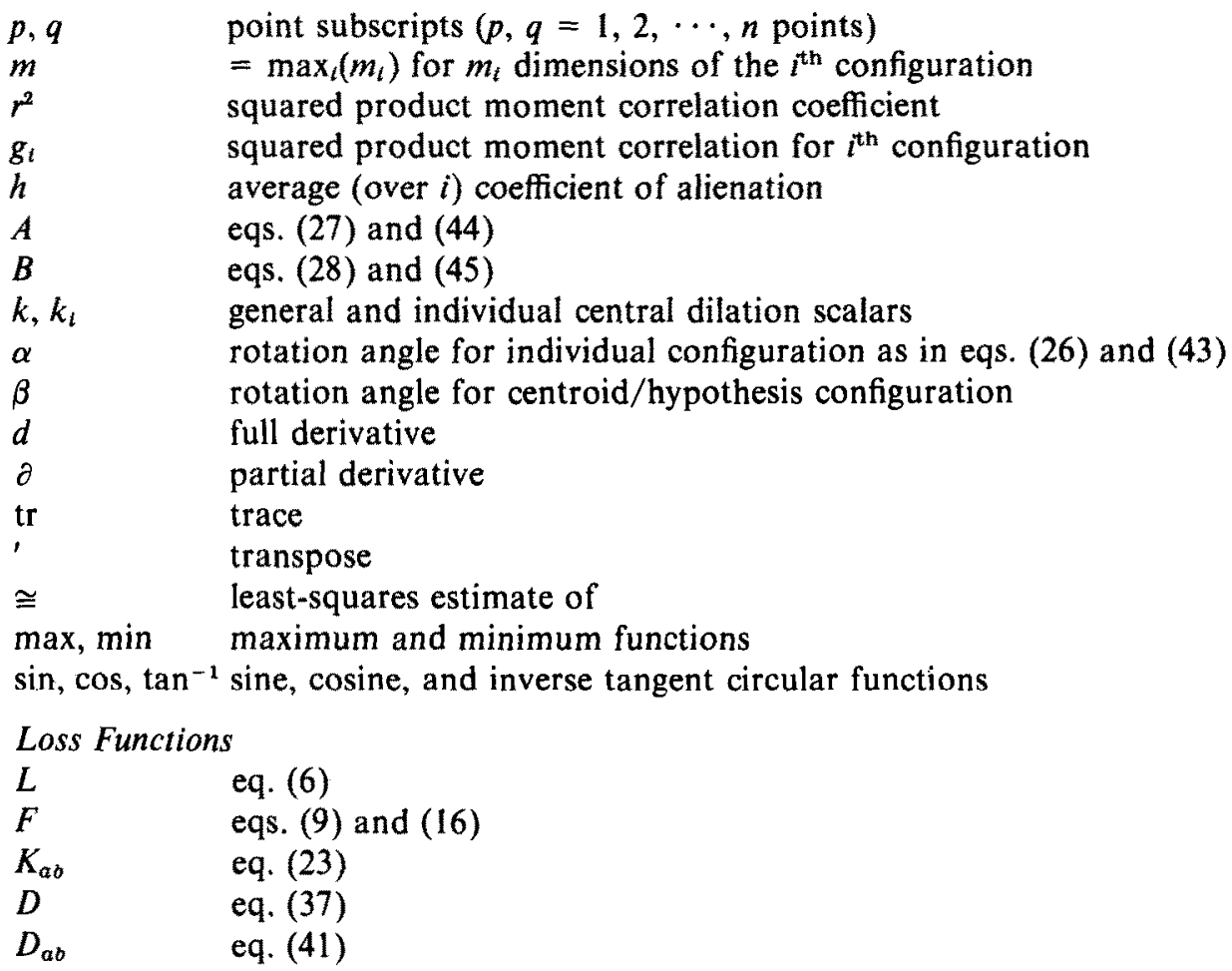

Matrices and Arrays

INDSCAL/IDIOSCAL

$G\left(g_{p a}\right) \quad$ group space

$C_{t}\left(c_{a}^{(i)}\right) \quad$ diagonal matrix of squared weights

$\hat{B}_{i}\left(\hat{b}_{p q}^{(i)}\right) \quad$ eq. (2)

$D_{l}\left(d_{p q}^{(i)}\right) \quad$ eq. (1)

$\hat{D}_{i}\left(d_{p q}^{(i)}\right) \quad$ eq. (1)

$T_{i}\left(t_{a b}^{(l)}\right) \quad$ eq. (5), an $m$-square orthonormal matrix

$\Lambda_{t}\left(\lambda_{a a}^{(i)}\right) \quad$ eq. (5), diagonal $m$-square matrix of squared weights

PINDIS

$X_{l}\left(x_{p a}^{(i)}\right) \quad$ rectangular coordinates of order $n \times m_{l}$ for configuration $i$

$\tilde{X}_{i}\left(\tilde{x}_{p a}^{(i)}\right) \quad X_{i}$ subjected to rigid motions and a central dilation as in eq. (10)

$\hat{X}_{l}\left(\hat{x}_{p a}^{(i)}\right) \quad$ optimally reflected $X_{t}$ as in eq. (45)

$X_{i}^{*}\left(x_{p a}^{*(i)}\right) \quad$ optimally rotated $X_{i} / \bar{X}_{i}$ as in eqs. (24) and (25)

$X_{i}^{u}\left(x_{p a}^{u(i)}\right) \quad$ optimally translated $X_{t} / \hat{X}_{l}$ as in eq. (50)

$Z\left(z_{p a}\right)$

$Z^{r}\left(z_{p a}^{r}\right)$

$Z_{i}^{r}\left(z_{p a}^{r(i)}\right)$

$Z^{t}\left(z_{p a}^{t}\right)$

$Z_{i}^{t}\left(z_{p a}^{t(t)}\right)$

$\bar{Z}\left(\bar{z}_{p a}\right)$

$Z^{*}\left(z_{p a}^{*}\right)$

$R_{l}\left(r_{a b}^{(i)}\right)$

$S_{i}\left(s_{a b}^{(i)}\right)$

$S\left(s_{a b}\right)$ rectangular coordinates of $n \times m$ centroid/hypothesis configuration optimally rotated $Z$ over all $i$ for dimension weighting optimally rotated $Z$ for each $i$ for dimension weighting optimally translated $Z$ over all $i$ for vector weighting optimally translated $Z$ for each $i$ for vector weighting the centroid of $Z_{i}^{r}$

eqs. (32) and (33) for dimension weighting rotations

$W_{i}\left(w_{a a}^{(i)}\right)$

$W^{*}\left(w_{a a}^{*(i)}\right)$ orthonormal matrix for orthogonally rotating any $X_{i}$ orthonormal matrix for rotating $Z$ orthogonally orthonormal matrix for rotating $\boldsymbol{Z}$ to $\bar{Z}$ eq. (19), the dimension weights applied to $Z^{r}$ eq. (21), the normalized $W_{i}$ 


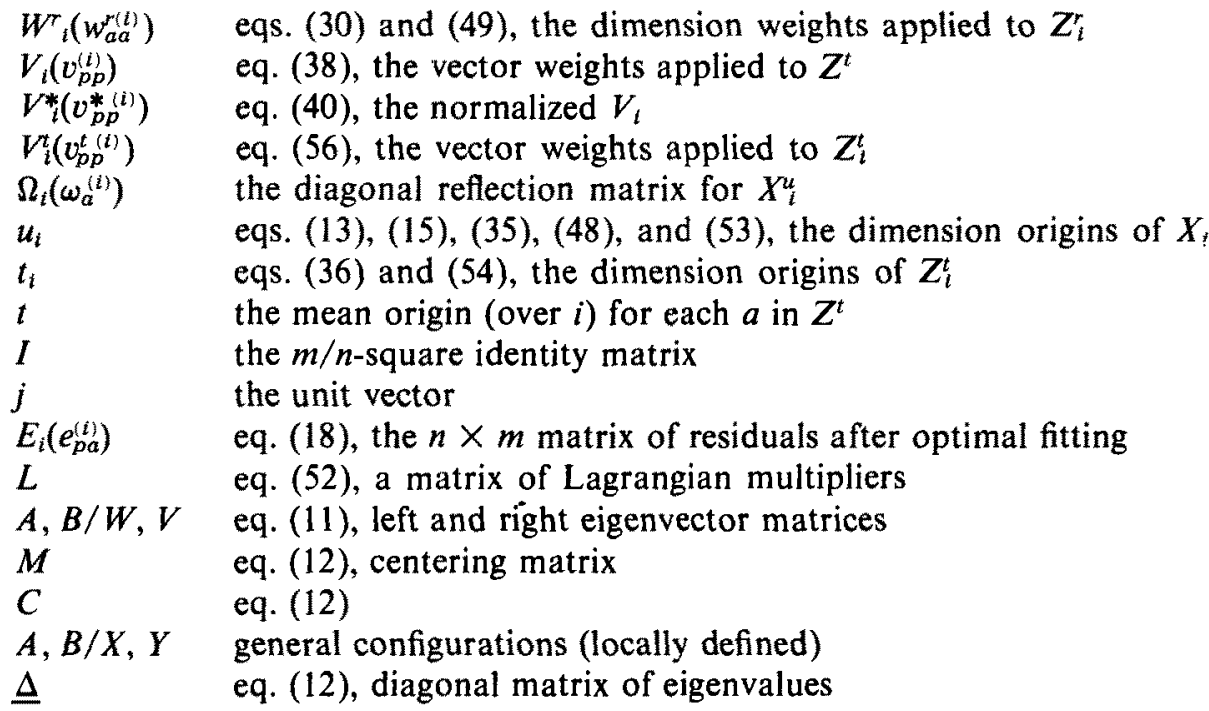

\section{REFERENCE NOTES}

1. Bloxom, B. Individual differences in multidimensional scaling (ETS RM 68-45). Princeton, N. J.: Educational Testing Service, 1968.

2. Borg, I. \& Lingoes, J. C. What weight should weights have in the analysis of individual differences? (Michigan Mathematical Psychology Program Tech. Rep. 76-5). Ann Arbor, Mich.: 1976.

3. Carroll, J. D. \& Chang, J. J. IDIOSCAL (Individual Differences In Orientation Scaling): A generalization of INDSCAL allowing idiosyncratic reference systems as well as an analytic approximation to INDSCAL. Paper presented at the Psychometric Society meetings, Princeton, N. J.: 1972.

4. Harshman, R. A. PARAFAC2: Mathematical and technical notes (UCLA Working Papers in Phonetics 22). L. A., Calif:: 1972.

5. Helm, C. E. A multidimensional ratio scaling analysis of color relations (Technical Report of Princeton University and the Educational Testing Service). Princeton, N. J.: 1959.

6. Lingoes, J. C. A neighborhood preserving transformation for fitting configurations. Paper presented at The U. S.-Japan Conference on Multidimensional Scaling, La Jolla, Calif.: 1975.

7. Schönemann, P. H., Carter, F. S., \& James, W. L. Contributions to subjective metrics scaling: I. COSPA, a fast method for fitting and testing Horan's model, and an empirical comparison with INDSCAL and ALSCAL (Krannert Graduate School of Management 587). W. Lafayette, Ind.: Purdue University, 1976.

8. Feger, H. Personal communication.

\section{REFERENCES}

Berge, Jos M. F. ten. Orthogonal procrustes rotation for two or more matrices. Psychometrika, 1977, 42, 267276.

Borg, I. Geometric representation of individual differences. In J. C. Lingoes (Ed.). Geometric representations of relational data. Ann Arbor, Mich.: Mathesis Press, 1977.

Borg, I. Ein Vergleich verschiedener Studien zur Lebensqualität. Zeitschrift für Sozialpsychologie, 1978 (in press).

Borg, 1. \& Lingoes, J. C. Ein direkter Transformationsansatz der multidimensionalen Analyse dreimodaler Datenmatrizen: Theorie und Anwendungen. Zeitschrift für Sozialpsychologie, 1977, 8, 98-114.

Carroll, J. D. \& Chang, J. J. Analysis of individual differences in multidimensional scaling via an $\mathrm{N}$-way generalization of "Eckart-Young" decomposition. Psychometrika, 1970, 35, 283-320.

Carroll, J. D. \& Wish, M. Models and methods for three-way multidimensional scaling. In D. H. Krantz, R. C. Atkinson, D, R. Luce, \& P. Suppes (Eds.), Contemporary developments in mathematical psychology (Vol. 2). San Francisco, Calif: W. H. Freeman, 1974.

Feger, H. Die Erfassung individueller Einstellungsstrukturen. Zeitschrift für Sozialpsychologie, 1974, 5, 57-105.

Feger, H. Längsschnittliche Erfassung intraindividueller Unterschiede bei Einstellungsstrukturen. In U. M. Lehr \& F. E. Weinert (Eds.). Entwicklung und Persönlichkeit. Stuttgart, W. Germany: Kohlhammer, 1975.

Fischer, G. H. \& Roppert, J. Ein Verfahren der Transformationsanalyse faktoren-analytischer Ergebnisse. In J. Roppert \& G. H. Fischer. Lineare Strukturen in Mathematik und Statistik. Wien, Austria: Physika Verlag, 1965. 
Gower, J. C. Generalized procrustean analysis. Psychometrika, 1975, 40, 33-51.

Guttman, L. A new approach to factor analysis: The radex. In P. F. Lazarsfeld (Ed.). Mathematical thinking in the social sciences. Glencoe, Ill: The Free Press, 1954.

Harman, H. H. Modern factor analysis. Chicago, Ill.: University of Chicago Press, 1967.

Helm, C. E. \& Tucker, L. R. Individual differences in the structure of color-perception. American Journal of Psychology, 1962, 75, 437-444.

Helm, C. E. Multidimensional ratio scaling analysis of perceived color relations. Journal of the Optical Society of America, 1964, 54, 256-262.

Horan, C. B. Multidimensional scaling: Combining observations when individuals have different perceptual structures. Psychometrika, 1969, 34, 139-165.

Kristof, W. \& Wingersky, B. Generalization of the orthogonal procrustes rotation procedure for more than two matrices. Proceedings of the 79th Annual Convention of the American Psychological Association, 1971, 89-90.

Levy, S. Use of the mapping sentence for coordinating theory and research: A cross-cultural example. Quality and Quantity, 1976, 10, 117-125.

Levy, S. \& Guttman, L. On the multivariate structure of well-being. Social Indicators Research, 1975, 2, 361-388.

Lingoes, J. C. The Guttman-Lingoes nonmetric program series. Ann Arbor, Mich.: Mathesis Press, 1973.

Lingoes, J. C. Progressively complex linear transformations for finding geometric similarities among data structures. In H. Hudson (Ed.). Classifying cultural and social data. San Francisco, Calif.: Jossey-Bass, 1978 (in press).

Lingoes, J. C. \& Schönemann, P. H. Alternative measures of fit for the Schönemann-Carroll matrix fitting algorithm. Psychometrika, 1974, 39, 423-427.

Lingoes, J. C. \& Borg, I. Procrustean indivdual differences scaling: PINDIS. Journal of Marketing Research, $1976,13,406-407$.

Lingoes, J. C. \& Borg, I. Procrustean individual differences scaling: PINDIS. Journal of Marketing Research, Sozialpsychologie, 1977, 8, 210-217.

Lissitz, R. W., Schönemann, P. H., \& Lingoes, J. C. A solution to the weighted procrustes problem in which the transformation is in agreement with the loss function. Psychometrika, 1976,41,547-550.

MacCallum, R. C. A comparison of two individual differences models for multidimensional scaling: Carroll and Chang's INDSCAL and Tucker's three-mode factor analysis. Unpublished doctoral dissertation, University of Illinois, Urbana, Illinois, 1974.

MacCallum, R. C. Effects of conditionality on INDSCAL and ALSCAL weights. Psychometrika, 1977, 42, 297305 .

Schönemann, P. H. A generalized solution of the orthogonal procrustes problem. Psychometrika, 1966, 31, 1-10.

Schönemann, P. H. \& Carroll, R. M. Fitting one matrix to another under choice of a central dilation and a rigid motion. Psychometrika, 1970, 35, 245-255.

Schönemann, P. H. An algebraic solution for a class of subjective metrics models. Psychometrika, 1972, 37, 441451.

Takane, Y, Young, F., \& de Leeuw, J. Nonmetric individual differences multidimensional scaling: An alternating least squares method with optimal scaling features. Psychometrika, 1977, 42, 7-67.

Torgerson, W. S. Theory and methods of scaling. New York, N. Y.: Wiley, 1958.

Tucker, L. R. Relations between multidimensional scaling and three-mode factor analysis. Psychometrika, 1972 , 37, 333-367.

Wish, M. \& Carroll, J. D. Applications of individual differences scaling to studies of human perception and judgement. In E. C. Carterette \& M. P. Friedman (Eds.). Handbook of perception (Vol. 2). New York, N. Y.: Academic Press, 1974.

Manuscript received $7 / 6 / 76$

First revision received $10 / 3 / 77$

Final version received $4 / 26 / 78$ 